Article

\title{
Pharmacological Properties and Chemical Profiles of Passiflora foetida L. Extracts: Novel Insights for Pharmaceuticals and Nutraceuticals
}

\author{
Annalisa Chiavaroli ${ }^{1}$, Simonetta Cristina Di Simone ${ }^{1}$, Kouadio Ibrahime Sinan ${ }^{2}$, \\ Maria Chiara Ciferri ${ }^{1}$, Giancarlo Angeles Flores ${ }^{3}$, Gokhan Zengin ${ }^{2, * \mathbb{D}}$, \\ Ouattara Katinan Etienne ${ }^{4}$, Gunes Ak ${ }^{2}$, Mohamad Fawzi Mahomoodally ${ }^{5,6}$, Sharmeen Jugreet ${ }^{6}$, \\ Zoltán Cziáky ${ }^{7}{ }^{\mathbb{D}}$, József Jekő ${ }^{7}$, Lucia Recinella ${ }^{1}$, Luigi Brunetti ${ }^{1}$, Sheila Leone ${ }^{1}$, \\ Paola Angelini ${ }^{3, *(D)}$, Roberto Venanzoni ${ }^{3}\left(\mathbb{D}\right.$, Luigi Menghini $^{1}\left(\mathbb{D}\right.$, Claudio Ferrante $^{1, *(D)}$ and \\ Giustino Orlando ${ }^{1}$
}

1 Department of Pharmacy, University “G. d'Annunzio" of Chieti-Pescara, 66100 Chieti, Italy; annalisa.chiavaroli@unich.it (A.C.); disimonesimonetta@gmail.com (S.C.D.S.); mariachiara.ciferri@outlook.it (M.C.C.); lucia.recinella@unich.it (L.R.); luigi.brunetti@unich.it (L.B.); sheila.leone@unich.it (S.L.); luigi.menghini@unich.it (L.M.); giustino.orlando@unich.it (G.O.)

2 Department of Biology, Faculty of Science, Selcuk University, Campus, Konya 42130, Turkey; sinankouadio@gmail.com (K.I.S.); akguneselcuk@gmail.com (G.A.)

3 Department of Chemistry, Biology and Biotechnology, University of Perugia, 06100 Perugia, Italy; giancarlo.angelesflores@studenti.unipg.it (G.A.F.); roberto.venanzoni@unipg.it (R.V.)

4 Laboratoire de Botanique, UFR Biosciences, Université Félix Houphouët-Boigny, Abidjan 00225, Cote D'Ivoire; katinan.etienne@gmail.com

5 Institute of Research and Development, Duy Tan University, Da Nang 550000, Vietnam; mohamad.fawzi.mahomoodally@tdtu.edu.vn

6 Department of Health Sciences, Faculty of Science, University of Mauritius, Réduit 230, Mauritius; sharmeenjugs@gmail.com

7 Agricultural and Molecular Research and Service Institute, University of Nyíregyháza, 4400 Nyíregyháza, Hungary; cziaky.zoltan@nye.hu (Z.C.); jjozsi@gmail.com (J.J.)

* Correspondence: gokhanzengin@selcuk.edu.tr (G.Z.); paola.angelini@unipg.it (P.A.); claudio.ferrante@unich.it (C.F.)

Received: 4 August 2020; Accepted: 19 August 2020; Published: 24 August 2020

\begin{abstract}
In the present study, Passiflora foetida extracts characterized by different polarities were studied for their phytochemical profile, enzyme inhibitory, and antioxidant potentials. In silico, in vitro and ex vivo studies were also carried out on methanol and water extracts for predicting pharmacokinetics and pharmacodynamics. In this regard, neuronal HypoE22 cells, isolated mouse skin tissues, and pathogen dermatophytes strains were exposed to extracts. Emphasis was given to the preventing effects induced by the extracts on hydrogen peroxide-induced alterations of prostaglandin $\mathrm{E}_{2}\left(\mathrm{PGE}_{2}\right), 1-\mathrm{dopa}$, and serotonin. Chemical analysis revealed the presence of similar compounds in infusion and methanolic extracts. The ex vivo studies also showed protective skin properties by P. foetida water and methanol extracts, as evidenced by the decrease of hydrogen peroxide-induced $\mathrm{PGE}_{2}$ level. Additionally, the blunting effects on hydrogen peroxide-induced l-dopa levels are consistent with the anti-tyrosinase effect exerted by both extracts. In silico studies demonstrated the affinity of extracts' phytochemicals, namely apigenin, chrysoeriol, loliolide, luteolin, quercetin, and vitexin, towards cyclo-oxygenase- 2 and tyrosinase. Finally, microbiological tests demonstrated the efficacy of P. foetida methanol and water extracts as anti-mycotic agents against Trichophyton and Arthroderma species, involved in skin inflammation. Hence, P. foetida L. extracts could represent potential sources of pharmaceuticals and nutraceuticals.
\end{abstract}


Keywords: Passiflora foetida; chemical profile; anti-oxidant/anti-inflammatory effects; neuroprotection; skin protection; bioinformatics/network pharmacology

\section{Introduction}

The genus Passiflora belongs to the Passifloraceae family and the members of this genus have a great interest in their therapeutic properties against anxiety and irritability [1]. Thus, the genus is significantly valuable for the development of novel drugs [2].

The biological activity of many medicinal plants and other natural products is directly related to the bioactive compounds they contain [3]. Consequently, many studies have focused on the analysis of the phytocompounds revealing the presence of alkaloids, phenols, glycosyl flavonoids, and cyanogenic compounds [4]. In earlier studies, the members of this genus contained important phytochemicals such as $\beta$-carbolines, harmala alkaloids, coumarins, maltol, phytosterols, and cyanogenic glycosides. Additionally, they have been reported to be rich in phenolic compounds, amino acid $\alpha$-alanine, and organic acids, including butyric, linoleic, formic, oleic, malic, linolenic, myristic, and palmitic acids [5] as well as d-fructose, d-glucose and raffinose [6].

Passiflora foetida L. popularly known as striking passionflower [7] is a particularly renowned species belonging to the genus Passiflora, with tremendous ethnobotanical applications. For instance, the decoction of leaves and fruits of P. foetida has been reported to treat asthma and biliousness, while the leaves and root decoction is employed as an emmenagogue and used in hysteria. Additionally, leaf paste is applied to the head for headache and giddiness. Besides, the herb is used in the form of poultices or lotions for erysipelas and skin diseases with inflammation [8]. P. foetida has also been described to treat anxiety, insomnia, sexual dysfunction, convulsion, cough as well as cancer [9].

Moreover, studies conducted on P. foetida have revealed extracts of the plant to possess numerous promising bioactivities such as antidiarrhoeal, antiulcerogenic, analgesic, antidepressant anti-inflammatory, anti-hypertensive, hepaprotective, anticancer, antibacterial and antinociceptive [8,10-17]. Similarly, several bioactive compounds isolated from $P$. foetida, especially flavonoids, have shown important pharmacological actions, such as luteolin and chrysoeriol that have been found to possess potent anti-inflammatory properties [18].

Although the existence of extensive documentation on the traditional uses of Passiflora species associated with a variety of health benefits, many species of the genus have still remained underexplored or have been modestly studied and thus require them to be scientifically validated, with $P$. foetida being one of them. Therefore, phytochemical profile, antioxidant, and enzyme inhibition activities were studied P. foetida extracts characterized by different polarities were studied. In silico, in vitro and ex vivo studies were also carried out on methanol and water extracts in order to predict pharmacokinetics and putative targets underlying traditional and innovative pharmacological applications of $P$. foetida. In this regard, the multidirectional pharmacological approach focused on the activities of the extracts as protective agents, on neuronal HypoE22 cells and isolated skin tissues, and antimicrobials against selected dermatophyte strains deeply involved in skin inflammation. Emphasis was given to the preventing effects induced by the extracts on the alterations of prostaglandin $\mathrm{E}_{2}\left(\mathrm{PGE}_{2}\right), 1-$ dopa, and serotonin levels following oxidative stress stimulus (hydrogen peroxide) challenging.

\section{Materials and Methods}

\subsection{Plant Material and Preparation of Extracts}

Passiflora foedita materials were collected in the neighborhood of Morofé in the city of Yamoussoukro (Lakes region, Ivory Coast) in 2019 by Kouadio Ibrahime Sinan. The plants were identified by Ouattara Katinan Etienne (Botanist at the Université Félix Houphouet Boigny, Ivory Coast). The plant samples 
were deposited in the Department of Biology at Selcuk University. The aerial parts (as mixed) were used. The plant materials were dried in shade for about 10 days and then grounded using a laboratory mill.

Maceration was used for sample preparation with ethyl acetate, methanol, methanol-water $(80 \%)$, and water as extraction solvents. For this purpose, $5 \mathrm{~g}$ of the samples were macerated with the solvents $(100 \mathrm{~mL})$ for $24 \mathrm{~h}$ at room temperature. After this, the mixtures were filtered and then the solvents were removed via a rotary evaporator. With respect to the infusion extract, $5 \mathrm{~g}$ of the plant material were infused with $100 \mathrm{~mL}$ boiled water. Thereafter, the water was dried using freeze-drying. All extracts were stored at $+4{ }^{\circ} \mathrm{C}$ until further studies.

\subsection{Profile of Bioactive Compounds}

The total phenolic and flavonoid contents in P. foetida extracts were determined by common spectrophotometric methods and the details for these methods were described in our previous paper [19]. Gallic acid (GAE, for phenolic, Sigma-Aldrich, St. Louis, MO, USA) and rutin (RE, for flavonoid, Sigma-Aldrich, St. Louis, MO, USA) were used as standards in the assays.

Gradient reversed-phase UHPLC separations with electrospray MS/MS detection (both positive and negative ion modes) were used for the structural characterization of the compounds presenting in different extracts. The UHPLC system consisted of the Dionex Ultimate 3000RS UHPLC (Waltham, MA, USA) instrument coupled to a Thermo Q Exactive Orbitrap mass spectrometer (Waltham, MA, USA). Chromatographic separation was achieved on a reversed-phase column Thermo Accucore C18 (100 mm $\times 2.1 \mathrm{~mm}$ i. d., $2.6 \mu \mathrm{m}$, Waltham, MA, USA) [20]. All analytical details were given in the Supplementary Materials.

\subsection{Determination of Antioxidant and Enzyme Inhibitory Effects}

Antioxidant properties of $P$. foetida extracts were tested by different methods. These methods included metal chelating, reducing power, and radical quenching assays. In these assays, standards were trolox (TE) and ethylenediaminetetraacetic acid (EDTA) [21]. Regarding the enzyme inhibition effects, we selected some enzymes including tyrosinase (EC1.14.18.1, from mushroom), cholinesterase (electric eel acetylcholinesterase (AChE) (type-VI-S), EC 3.1.1.7; horse serum butyrylcholinesterase (BChE) (EC 3.1.1.8)), $\alpha$-amylase (EC. 3.2.1.1, from porcine pancreas), and $\alpha$-glucosidase (EC. 3.2.1.20, from Saccharomyces cerevisiae). In enzyme inhibitory assays, standards were kojic acid (KAE), galantamine (GALAE), and acarbose (ACAE). Both antioxidant and enzyme inhibitory results were expressed as standard equivalents. All reagents, enzymes and standards were purchased from Sigma-Aldrich, St. Louis, MO, USA

\subsection{Brine Shrimp (Artemia salina) Lethality Test}

Brine shrimp (Artemia salina) cysts were cultured in oxygenated artificial water, as previously reported [22]. After $24 \mathrm{~h}$, brine shrimp larvae were gently transferred with a pipette in 6 well plates containing $2 \mathrm{~mL}$ of $P$. foetida extracts at different concentrations $(0.1-20 \mathrm{mg} / \mathrm{mL})$ in artificial seawater. Ten larvae per well were incubated at $25-28{ }^{\circ} \mathrm{C}$ for $24 \mathrm{~h}$. After $24 \mathrm{~h}$, the number of living nauplii was counted under a light microscope and compared to the control untreated group. Results were expressed as a percentage of mortality calculated as: $((\mathrm{T}-\mathrm{S}) / \mathrm{T}) \times 100$. $\mathrm{T}$ is the total number of incubated larvae and $S$ is the number of survival nauplii. Living nauplii were considered those exhibiting light activating movements during $10 \mathrm{~s}$ of observation. For each experimental condition, two replicates per plate were performed and experimental triplicates were performed in separate plates. Experiments were conducted in triplicates. Results of the eco-toxicological test were expressed as percentage lethality.

\subsection{Cell Culture}

The anti-proliferative effect of $P$. foetida extracts was evaluated in rat hypothalamus HypoE22 cells and cultured in DMEM supplemented with $10 \%(v / v)$ heat-inactivated fetal bovine serum and penicillin-streptomycin $(100 \mu \mathrm{g} / \mathrm{mL})$ (all from EuroClone SpA Life-Sciences-Division, Milano, 
Italy) [22]. The Hypo-E22 cell line (Catalogue Number: CLU213) was purchased from Cedarlane Corporation (Burlington, ON, Canada). Cells were grown at $37^{\circ} \mathrm{C}$ in a humified atmosphere of $5 \% \mathrm{CO}_{2}$. When indicated, the cells were treated with $\mathrm{H}_{2} \mathrm{O}_{2} 300 \mu \mathrm{M}$ for $3 \mathrm{~h}$ and different concentrations of $P$. foetida extracts $(10-500 \mu \mathrm{g} / \mathrm{mL})$. The cell viability was evaluated after $24 \mathrm{~h}$ of culture by MTT (3-[4,5-dimethyl-thiazol-2-yl]-2,5-diphenyl tetrazolium bromide) growth assay (Sigma-Aldrich, St. Louis, MO, USA), based on the capability of viable cells to reduce MTT into a colored formazan product. The cells were seeded into 96-well plates at $5 \times 103$ cells/well. At the established time point, the medium was replaced with a fresh one containing $0.5 \mathrm{mg} / \mathrm{mL} \mathrm{MTT}$, and the cells were incubated for $3 \mathrm{~h}$ at $37^{\circ} \mathrm{C}$. After a further incubation of the samples in DMSO for $30 \mathrm{~min}$ at $37^{\circ} \mathrm{C}$, the absorbance at $570 \mathrm{~nm}$ was measured using a Multiscan GO microplate spectrophotometer (Thermo Fisher Scientific, Waltham, MA, USA). The values obtained in the absence of cells were considered as background and subtracted from the optical density values of the samples. Three independent experiments were performed under the same experimental conditions. The wound healing test was employed to determine the role of the extracts on spontaneous cell migration. The detailed paradigm is included in our previous paper [22].

\subsection{Ex Vivo Model of LPS-Induced Toxicity in Isolated Mouse Skin Tissue}

Skin specimens were collected from male adult C57BL6 mice sacrificed by $\mathrm{CO}_{2}$ inhalation. The experimental paradigm obtained the approval from the Italian Ministry of Health (Authorization Number: F4738.N.5QP, released on 8 February 2020). Skin specimens were stimulated with water and methanol $P$. foetida extracts $(10-500 \mu \mathrm{g} / \mathrm{mL})$. PGE $_{2}$ level (pg/mg wet tissue) was measured by radioimmunoassay (RIA) [23] in tissue supernatants.

\subsection{HPLC Analysis}

Cell and tissue supernatants were analyzed through high-performance liquid chromatography (HPLC) coupled to electrochemical detection for the determination of 5-HT and l-dopa levels respectively. The detailed protocol related to 1-dopa and 5-HT identification and quantification is included in a previous article of ours [24].

\subsection{Antifungal Activity of the Extract}

The antifungal activity of $P$. foetida extracts was evaluated against multiple dermatophytes species, namely Trichophyton rubrum (CCF 4933), T. rubrum (CCF 4879), T. rubrum (CCF 4834), T. mentagrophytes (CCF 4823), T. mentagrophytes (CCF 5930), Arthroderma crocatum (CCF 5300), A. curreyi (CCF 5207), A. gypseum (CCF 6261), A. insingulare (CCF 5417), A. quadrifidum (CCF 5792). The experimental procedure was reported in our previous paper [22].

\subsection{Bioinformatics and Docking Studies}

The bioinformatics study was carried out according to the protocol published by Gu et al. [25]. The canonical SMILES were run by the SwissTargetPrediction and SwissADME platforms, for in silico prediction of putative targets and pharmacokinetic profile. Additionally, the prediction of phytochemicals' toxicity was conducted through the "Toxicity Estimation Software Tool" (T.E.S.T.) developed by the United States Environmental Protection Agency (EPA). The identification of target proteins was confirmed by the UniProt database (https:/www.uniprot.org/). Subsequently, a components-targets analysis was conducted through Cytoscape software (3.7.2 version). Docking calculations were carried out as previously reported [26]. The Protein Data Bank (PDB) numbers of each docked proteins were: $5 \mathrm{M} 8 \mathrm{P}$ for the tyrosinase; $1 \mathrm{CX} 2$ for the cyclo-oxygenase (COX)-2 enzyme; 2Z5X for the monoamine oxygenase-A (MAO-A) enzyme; 1GQR for the acetylcholinesterase (AchE) enzyme. 


\subsection{Statistical Analysis}

Data were means \pm S.D. of three experiments conducted in triplicate and analyzed by analysis of variance (ANOVA) coupled to Tukey post hoc test. Statistical significance was set at $p<0.05$, and GraphPad Prism version 5.01 for Windows (GraphPad Software, San Diego, CA, USA).

\section{Results and Discussion}

In the present study, the quantitative analysis indicated the total phenolic and flavonoid contents of the tested extracts ranging from $22.28-36.91$ and $3.22-50.11 \mathrm{mg} / \mathrm{g}$ respectively. While the ethyl acetate extract contained the highest phenolic content $(39.61 \pm 0.26 \mathrm{mg} / \mathrm{g})$, the methanolic extract $(80 \%)$ was found to yield the highest flavonoid content $(50.11 \pm 0.78 \mathrm{mg} / \mathrm{g})$ compared to the other extracts, thus indicating that ethyl acetate and methanolic $(80 \%)$ as effective solvents to extract phenolics and flavonoids from $P$. foetida, respectively. On the other hand, water maceration and infusion extracts contained the lowest TFC ( $3.22 \pm 0.45$ and $4.27 \pm 0.45 \mathrm{mg} / \mathrm{g}$, respectively) (Table 1). Polar solvents are frequently used for recovering polyphenols from plant matrices. The most suitable solvents are aqueous mixtures containing ethanol, methanol, acetone, and ethyl acetate [23]. This is consistent, albeit partially, with the present findings. In fact, it is recognized that one of the most crucial factors affecting the extraction efficiency of bioactive compounds from plant materials and their resulting health benefits is the extraction solvent [27].

Table 1. Total phenolic and flavonoid contents of the tested extracts.

\begin{tabular}{ccc}
\hline Extracts & TPC $(\mathbf{m g ~ G A E} / \mathbf{g})$ & TFC $(\mathbf{m g}$ RE/g) \\
\hline EA & $39.61 \pm 0.26^{\mathrm{a}}$ & $14.50 \pm 0.54^{\mathrm{b}}$ \\
$\mathrm{MeOH}$ & $24.59 \pm 0.24^{\mathrm{b}}$ & $10.52 \pm 0.59^{\mathrm{c}}$ \\
MeOH $(80 \%)$ & $21.30 \pm 0.20^{\mathrm{d}}$ & $50.11 \pm 0.78^{\mathrm{a}}$ \\
Water maceration & $22.18 \pm 0.16^{\mathrm{c}}$ & $3.22 \pm 0.45^{\mathrm{d}}$ \\
Infusion & $24.24 \pm 0.51^{\mathrm{b}}$ & $4.27 \pm 0.45^{\mathrm{d}}$
\end{tabular}

Values are reported as mean \pm S.D. EA: Ethyl acetate; MeOH: methanolic; TPC: Total phenolic content; TFC: Total flavonoid content; GAE: Gallic acid equivalent; RE: Rutin equivalent. Different letters (a, b, c and d) indicate significant differences in the extracts $(p<0.05)$.

The total flavonoid and phenolic contents of P. foetida extracts have also been studied [28-30]. Notably, in one study, it was found that the flavonoid and phenolic contents were higher in aqueous and ethanolic extracts of $P$. foetida leaf and root, whereas lesser amounts were present in petroleum ether extracts [28].

Remarkably, the phytochemical analysis of methanolic and infusion extracts revealed the same 47 compounds to be present in both extracts (Tables 2 and 3). However, methanolic extract was found to contain an additional compound, namely isorhamnetin-O-hexoside. The extracts were found to contain miscellaneous mixtures of compounds composed of flavones, flavonols, and their derivatives including flavones glycosides and hexosides, amongst others. In fact, flavonoids, especially their glycosides, are the most vital phytochemicals in diets and are of great general interest owing to their diverse bioactivity. Natural flavonoids mostly exist as their O-glycoside or C-glycoside forms in plants [31]. Some examples of flavonoid O-glycosides identified in the extracts herein were isoquercitrin and isorhamnetin-3-O-glucoside. However, the presence of flavonoid C-glycosides was mostly dominant in the extracts. Examples included vitexin, isovitexin, orientin, and isoorientin, among others. Other Passiflora species have also been reported to be good sources of bioactive flavonoid C-glycosides [32,33]. In the study of Zucolotto et al. [32], the C-glycosyl flavonoids profile of leaf and pericarp extracts of South American Passiflora species were investigated. Although the different species and varieties showed different major constituents, the C-glycosyl flavonoids identified more frequently were orientin, isoorientin, vitexin, and isovitexin. This is in accordance with the findings of the present study. Paradoxically, it was noted that the chemical composition identified in the methanolic extract of P. foetida herein differed significantly from the one obtained in the study of Sisin et al. [29]. 
Table 2. Chemical profile of infusion extract

\begin{tabular}{|c|c|c|c|c|c|c|c|c|c|c|c|c|}
\hline No. & Name & Formula & Rt & $(\mathrm{M}+\mathrm{H})^{+}$ & $(\mathrm{M}-\mathrm{H})^{-}$ & Fragment 1 & Fragment 2 & Fragment 3 & Fragment 4 & Fragment 5 & Relative Ratio (\%) & Literature \\
\hline 1 & Pantothenic acid & C9H17NO5 & 7.24 & 220.11850 & & 202.1077 & 184.0972 & 116.0346 & 90.0555 & 72.0451 & 2.16 & \\
\hline 2 & Kynurenic acid & $\mathrm{C} 10 \mathrm{H} 7 \mathrm{NO} 3$ & 14.18 & 190.05042 & & 162.0551 & 144.0448 & 116.0501 & 89.0393 & & 0.40 & [34] \\
\hline 3 & Dihydroxycoumarin & $\mathrm{C} 9 \mathrm{H} 6 \mathrm{O} 4$ & 14.92 & 179.03444 & & 147.0442 & 135.0443 & 133.0287 & 123.0443 & 105.0338 & 0.20 & \\
\hline 4 & Naringenin-6,8-di-C-glucoside & $\mathrm{C} 27 \mathrm{H} 32 \mathrm{O} 15$ & 17.38 & & 595.16630 & 577.1575 & 505.1364 & 475.1267 & 385.0935 & 355.0831 & 0.08 & \\
\hline 5 & Luteolin-di-C-hexoside & $\mathrm{C} 27 \mathrm{H} 30 \mathrm{O} 16$ & 18.36 & 611.16121 & & 593.1509 & 575.1404 & 473.1085 & 341.0658 & 311.0552 & 0.19 & \\
\hline 6 & Phaselic acid (2-O-Caffeoylmalic acid) & $\mathrm{C} 13 \mathrm{H} 12 \mathrm{O} 8$ & 18.73 & & 295.04540 & 179.0342 & 135.0441 & 133.0131 & 115.0024 & 71.0123 & 0.37 & \\
\hline 7 & $\begin{array}{l}\text { Isololiolide } \\
\text { a }\end{array}$ & $\mathrm{C} 11 \mathrm{H} 16 \mathrm{O} 3$ & 18.78 & 197.11777 & & 179.1069 & 161.0963 & 135.1171 & 133.1015 & 107.0861 & 0.82 & \\
\hline 8 & Vicenin-2 (Apigenin-6,8-di-C-glucoside) & $\mathrm{C} 27 \mathrm{H} 30 \mathrm{O} 15$ & 19.36 & 595.16630 & & 559.1451 & 541.1334 & 457.1133 & 325.0706 & 295.0601 & 7.26 & \\
\hline 9 & Apigenin-C-hexoside-C-pentoside isomer 1 & $\mathrm{C} 26 \mathrm{H} 28 \mathrm{O} 14$ & 19.78 & 565.15574 & & 547.1455 & 529.1345 & 379.0815 & 325.0708 & 295.0605 & 0.19 & \\
\hline 10 & Luteolin-C-hexoside-C-pentoside & $\mathrm{C} 26 \mathrm{H} 28 \mathrm{O} 15$ & 20.03 & 581.15065 & & 563.1394 & 545.1295 & 395.0762 & 341.0656 & 311.0549 & 0.09 & \\
\hline 11 & Loliolide & $\mathrm{C} 11 \mathrm{H} 16 \mathrm{O} 3$ & 20.08 & 197.11777 & & 179.1069 & 161.0964 & 135.1172 & 133.1015 & 107.0860 & 4.46 & \\
\hline 12 & Apigenin-C-hexoside-C-pentoside isomer 2 & $\mathrm{C} 26 \mathrm{H} 28 \mathrm{O} 14$ & 20.46 & 565.15574 & & 529.1345 & 511.1239 & 427.1031 & 409.0923 & 295.0605 & 0.96 & \\
\hline 13 & Apigenin-C-hexoside-C-pentoside isomer 3 & $\mathrm{C} 26 \mathrm{H} 28 \mathrm{O} 14$ & 20.73 & 565.15574 & & 529.1350 & 511.1242 & 427.1031 & 409.0921 & 295.0603 & 1.04 & \\
\hline 14 & Orientin (Luteolin-8-C-glucoside) & $\mathrm{C} 21 \mathrm{H} 20 \mathrm{O} 11$ & 20.86 & 449.10839 & & 431.0979 & 413.0873 & 353.0660 & 329.0658 & 299.0555 & 0.29 & [18] \\
\hline 15 & Apigenin-C-hexoside-C-pentoside isomer 4 & $\mathrm{C} 26 \mathrm{H} 28 \mathrm{O} 14$ & 21.11 & 565.15574 & & 529.1350 & 469.1136 & 379.0816 & 325.0710 & 295.0603 & 6.32 & \\
\hline 16 & Isoorientin (Luteolin-6-C-glucoside) & $\mathrm{C} 21 \mathrm{H} 20 \mathrm{O} 11$ & 21.19 & 449.10839 & & 431.0973 & 413.0872 & 353.0659 & 329.0659 & 299.0553 & 1.59 & \\
\hline 17 & Luteolin-C-hexoside & $\mathrm{C} 21 \mathrm{H} 20 \mathrm{O} 11$ & 21.56 & 449.10839 & & 431.0974 & 413.0871 & 353.0658 & 329.0657 & 299.0553 & 0.10 & \\
\hline $18^{1}$ & Vitexin (Apigenin-8-C-glucoside) & $\mathrm{C} 21 \mathrm{H} 20 \mathrm{O} 10$ & 21.84 & 433.11347 & & 415.1031 & 397.0921 & 379.0812 & 313.0709 & 283.0605 & 11.58 & [18] \\
\hline 19 & Apigenin-C-hexoside-C-pentoside isomer 5 & $\mathrm{C} 26 \mathrm{H} 28 \mathrm{O} 14$ & 22.11 & 565.15574 & & 529.1349 & 397.0924 & 379.0814 & 325.0710 & 295.0605 & 0.38 & \\
\hline $20^{1}$ & Vitexin-2"-O-rhamnoside & $\mathrm{C} 27 \mathrm{H} 30014$ & 22.18 & 579.17138 & & 433.1133 & 415.1025 & 397.0922 & 313.0708 & 283.0603 & 5.69 & \\
\hline 21 & Apigenin-C-hexoside-C-pentoside isomer 6 & $\mathrm{C} 26 \mathrm{H} 28 \mathrm{O} 14$ & 22.39 & 565.15574 & & 529.1348 & 397.0922 & 379.0815 & 325.0708 & 295.0605 & 0.49 & \\
\hline 22 & Methoxy-tetrahydroxy(iso)flavone-O-hexoside isomer 1 & $\mathrm{C} 22 \mathrm{H} 22 \mathrm{O} 12$ & 22.47 & & 477.10331 & 462.0813 & 315.0519 & 300.0281 & 299.0200 & 271.0254 & 0.06 & \\
\hline 23 & Apigenin-C-hexoside-C-pentoside isomer 7 & $\mathrm{C} 26 \mathrm{H} 28 \mathrm{O} 14$ & 22.61 & 565.15574 & & 529.1346 & 379.0814 & 337.0710 & 325.0706 & 295.0603 & 0.36 & \\
\hline 24 & Isovitexin (Apigenin-6-C-glucoside) & $\mathrm{C} 21 \mathrm{H} 20 \mathrm{O} 10$ & 22.74 & 433.11347 & & 415.1024 & 397.0921 & 379.0812 & 313.0708 & 283.0602 & 30.95 & \\
\hline 25 & Isoscoparin or Scoparin & $\mathrm{C} 22 \mathrm{H} 22 \mathrm{O} 11$ & 23.17 & 463.12404 & & 445.1131 & 427.1027 & 367.0813 & 343.0813 & 313.0707 & 0.20 & \\
\hline 26 & Methoxy-tetrahydroxy(iso)flavone-O-hexoside isomer 2 & $\mathrm{C} 22 \mathrm{H} 22 \mathrm{O} 12$ & 23.35 & & 477.10331 & 462.0819 & 315.0518 & 301.0360 & 299.0202 & 271.0253 & 0.02 & \\
\hline $27^{1}$ & Isoquercitrin (Quercetin-3-O-glucoside) & $\mathrm{C} 21 \mathrm{H} 20 \mathrm{O} 12$ & 23.47 & & 463.08765 & 301.0359 & 300.0280 & 271.0254 & 255.0301 & 178.9978 & 0.19 & \\
\hline $28^{1}$ & $\begin{array}{l}\text { Isorhamnetin-3-O-glucoside } \\
\text { Istide }\end{array}$ & $\mathrm{C} 22 \mathrm{H} 22 \mathrm{O} 12$ & 25.48 & & 477.10330 & 315.0521 & 314.0440 & 285.0411 & 271.0252 & 243.0299 & 0.23 & \\
\hline 29 & Abscisic acid & $\mathrm{C} 15 \mathrm{H} 20 \mathrm{O} 4$ & 25.81 & & 263.12834 & 219.1388 & 204.1151 & 201.1280 & 152.0832 & 151.0754 & 0.14 & \\
\hline 30 & Dihydroxy(iso)flavone-C-hexoside & C21H20O9 & 26.18 & 417.11856 & & 399.1079 & 351.0865 & 321.0759 & 297.0759 & 267.0653 & 0.16 & \\
\hline 31 & Methoxy-pentahydroxy(iso)flavone isomer 1 & $\mathrm{C} 16 \mathrm{H} 12 \mathrm{O} 8$ & 26.34 & & 331.04540 & 316.0229 & 287.0204 & 271.0254 & 270.0171 & 259.0248 & 0.24 & \\
\hline 32 & Methoxy-tetrahydroxy(iso)flavone-O-hexoside isomer 3 & $\mathrm{C} 22 \mathrm{H} 22 \mathrm{O} 12$ & 27.18 & & 477.10331 & 462.0805 & 315.0517 & 300.0281 & 299.0202 & 271.0254 & 0.05 & \\
\hline 33 & $\begin{array}{l}\text { Methoxy-pentahydroxy(iso)flavone isomer } 2 \\
\text { Met }\end{array}$ & $\mathrm{C} 16 \mathrm{H} 12 \mathrm{O} 8$ & 27.53 & & 331.04540 & 316.0229 & 287.0203 & 271.0248 & 270.0172 & 259.0243 & 0.13 & \\
\hline $34^{1}$ & Quercetin $\left(3,3^{\prime}, 4^{\prime}, 5,7-\right.$ Pentahydroxyflavone) & $\mathrm{C} 15 \mathrm{H} 10 \mathrm{O} 7$ & 27.57 & & 301.03483 & 273.0402 & 178.9978 & 151.0026 & 121.0282 & 107.0125 & 0.14 & \\
\hline $35^{1}$ & Naringenin $\left(4^{\prime}, 5,7\right.$-Trihydroxyflavanone) & $\mathrm{C} 15 \mathrm{H} 12 \mathrm{O} 5$ & 27.75 & & 271.06065 & 227.0706 & 177.0186 & 151.0026 & 119.0489 & 107.0125 & 0.15 & \\
\hline 36 & Methoxy-pentahydroxy(iso)flavone isomer 3 & $\mathrm{C} 16 \mathrm{H} 12 \mathrm{O} 8$ & 27.78 & & 331.04540 & 316.0227 & 299.0202 & 287.0216 & 271.0253 & 259.0256 & 0.55 & \\
\hline 37 & Trihydroxy-trimethoxy(iso)flavone-O hexoside & $\mathrm{C} 24 \mathrm{H} 26 \mathrm{O} 13$ & 27.95 & & 521.12952 & 506.1082 & 359.0779 & 358.0700 & 344.0544 & 329.0309 & 0.44 & \\
\hline $38^{1}$ & $\begin{array}{l}\text { Luteolin }\left(3^{\prime}, 4^{\prime}, 5,7 \text {-Tetrahydroxyflavone) }\right. \\
\text { (a) }\end{array}$ & $\mathrm{C} 15 \mathrm{H} 10 \mathrm{OO}$ & 28.44 & & 285.03991 & 217.0500 & 199.0397 & 175.0394 & 151.0026 & 133.0283 & 1.91 & [18] \\
\hline 39 & Quercetin-4'-O-methyl ether & $\mathrm{C} 16 \mathrm{H} 12 \mathrm{O} 7$ & 28.79 & & 315.05048 & 300.0280 & 271.0252 & 255.0301 & 243.0298 & 227.0344 & 6.71 & [18] \\
\hline 40 & Apigenin $\left(4^{\prime}, 5,7\right.$-Trihydroxyflavone $)$ & $\mathrm{C} 15 \mathrm{H} 10 \mathrm{O} 5$ & 30.28 & & 269.04500 & 227.0352 & 225.0555 & 151.0027 & 149.0232 & 117.0332 & 1.38 & [18] \\
\hline 41 & Chrysoeriol (3'-Methoxy-4',5,7-trihydroxyflavone) & $\mathrm{C} 16 \mathrm{H} 12 \mathrm{O} 6$ & 30.57 & & 299.05556 & 284.0331 & 256.0379 & 227.0347 & 151.0025 & 107.0123 & 0.60 & [18] \\
\hline 42 & Dimethoxy-trihydroxy(iso)flavone isomer 1 & $\mathrm{C} 17 \mathrm{H} 14 \mathrm{O} 7$ & 31.11 & & 329.06613 & 314.0439 & 299.0202 & 285.0413 & 271.0254 & 243.0300 & 5.51 & \\
\hline 43 & Dihydroxy-dimethoxy(iso)flavone isomer 1 & C17H14O6 & 32.37 & & 313.07122 & 298.0484 & 297.0409 & 283.0251 & 269.0465 & 255.0298 & 0.01 & [35] \\
\hline 44 & Dihydroxy-dimethoxy(iso)flavone isomer 2 & C17H14O6 & 32.92 & & 313.07122 & 298.0483 & 297.0409 & 283.0252 & 269.0467 & 255.0291 & 0.03 & [35] \\
\hline 45 & Dimethoxy-trihydroxy(iso)flavone isomer 2 & $\mathrm{C} 17 \mathrm{H} 14 \mathrm{O} 7$ & 33.29 & & 329.06613 & 314.0439 & 299.0202 & 285.0402 & 271.0254 & 243.0306 & 5.12 & \\
\hline 46 & Dihydroxy-dimethoxy(iso)flavone isomer 3 & C17H14O6 & 34.80 & & 313.07122 & 298.0487 & 283.0252 & 270.0541 & 269.0455 & 255.0300 & 0.03 & \\
\hline 47 & Dihydroxy-dimethoxy(iso)flavone isomer 4 & C17H14O6 & 35.48 & & 313.07122 & 298.0487 & 283.0253 & 269.0460 & 255.0301 & 242.0219 & 0.06 & [35] \\
\hline
\end{tabular}

${ }^{1}$ Confirmed by standard. 
Table 3. Chemical profile of methanolic extract.

\begin{tabular}{|c|c|c|c|c|c|c|c|c|c|c|c|c|}
\hline No. & Name & Formula & Rt & $(\mathrm{M}+\mathrm{H})^{+}$ & $(\mathrm{M}-\mathrm{H})^{-}$ & Fragment 1 & Fragment 2 & Fragment 3 & Fragment 4 & Fragment 5 & Relative Ratio (\%) & Literature \\
\hline 1 & Pantothenic acid & C9H17NO5 & 7.39 & 220.11850 & & 202.1077 & 184.0973 & 116.0347 & 90.0556 & 72.0452 & 1.72 & \\
\hline 2 & Kynurenic acid & $\mathrm{C} 10 \mathrm{H} 7 \mathrm{NO} 3$ & 14.21 & 190.05042 & & 162.0551 & 144.0447 & 116.0497 & 89.0392 & & 0.36 & [34] \\
\hline 3 & Dihydroxycoumarin & $\mathrm{C} 9 \mathrm{H} 6 \mathrm{O} 4$ & 14.92 & 179.03444 & & 147.0442 & 135.0444 & 133.0287 & 123.0444 & 105.0338 & 0.18 & \\
\hline 4 & Naringenin-6,8-di-C-glucoside & $\mathrm{C} 27 \mathrm{H} 32 \mathrm{O} 15$ & 17.38 & & 595.16630 & 577.1563 & 505.1341 & 475.1252 & 385.0934 & 355.0828 & 0.07 & \\
\hline 5 & Luteolin-di-C-hexoside & $\mathrm{C} 27 \mathrm{H} 30 \mathrm{O} 16$ & 18.39 & 611.16121 & & 593.1504 & 575.1391 & 473.1087 & 341.0659 & 311.0553 & 0.11 & \\
\hline 6 & Phaselic acid (2-O-Caffeoylmalic acid) & $\mathrm{C} 13 \mathrm{H} 12 \mathrm{O} 8$ & 18.74 & & 295.04540 & 179.0342 & 135.0440 & 133.0131 & 115.0024 & 71.0123 & 0.14 & \\
\hline 7 & Isololiolide & $\mathrm{C} 11 \mathrm{H} 16 \mathrm{O} 3$ & 18.80 & 197.11777 & & 179.1070 & 161.0962 & 135.1171 & 133.1015 & 107.0860 & 0.83 & \\
\hline 8 & Vicenin-2 (Apigenin-6,8-di-C-glucoside) & C27H30O15 & 19.38 & 595.16630 & & 559.1458 & 541.1343 & 457.1138 & 325.0709 & 295.0604 & 4.91 & \\
\hline 9 & Apigenin-C-hexoside-C-pentoside isomer 1 & $\mathrm{C} 26 \mathrm{H} 28 \mathrm{O} 14$ & 19.79 & 565.15574 & & 547.1466 & 529.1346 & 379.0814 & 325.0710 & 295.0606 & 0.14 & \\
\hline 10 & Luteolin-C-hexoside-C-pentoside & $\mathrm{C} 26 \mathrm{H} 28 \mathrm{O} 15$ & 20.05 & 581.15065 & & 563.1425 & 545.1283 & 395.0771 & 341.0663 & 311.0553 & 0.05 & \\
\hline 11 & Loliolide & $\mathrm{C} 11 \mathrm{H} 16 \mathrm{O} 3$ & 20.09 & 197.11777 & & 179.1070 & 161.0963 & 135.1172 & 133.1015 & 107.0860 & 4.84 & \\
\hline 12 & Apigenin-C-hexoside-C-pentoside isomer 2 & $\mathrm{C} 26 \mathrm{H} 28 \mathrm{O} 14$ & 20.48 & 565.15574 & & 529.1347 & 511.1249 & 427.1035 & 409.0924 & 295.0607 & 0.73 & \\
\hline 13 & Apigenin-C-hexoside-C-pentoside isomer 3 & $\mathrm{C} 26 \mathrm{H} 28 \mathrm{O} 14$ & 20.75 & 565.15574 & & 529.1352 & 511.1248 & 427.1032 & 409.0924 & 295.0602 & 0.73 & \\
\hline 14 & Orientin (Luteolin-8-C-glucoside) & $\mathrm{C} 21 \mathrm{H} 20 \mathrm{O} 11$ & 20.87 & 449.10839 & & 431.0981 & 413.0876 & 353.0660 & 329.0660 & 299.0553 & 0.26 & [18] \\
\hline 15 & Apigenin-C-hexoside-C-pentoside isomer 4 & $\mathrm{C} 26 \mathrm{H} 28 \mathrm{O} 14$ & 21.12 & 565.15574 & & 529.1350 & 469.1142 & 379.0815 & 325.0710 & 295.0605 & 4.32 & {$[10]$} \\
\hline 16 & Isoorientin (Luteolin-6-C-glucoside) & $\mathrm{C} 21 \mathrm{H} 20 \mathrm{O} 11$ & 21.21 & 449.10839 & & 431.0982 & 413.0872 & 353.0660 & 329.0660 & 299.0553 & 1.52 & \\
\hline 17 & Luteolin-C-hexoside & $\mathrm{C} 21 \mathrm{H} 20 \mathrm{O} 11$ & 21.57 & 449.10839 & & 431.0982 & 413.0872 & 353.0661 & 329.0660 & 299.0554 & 0.33 & \\
\hline $18^{1}$ & Vitexin (Apigenin-8-C-glucoside) & $\mathrm{C} 21 \mathrm{H} 20 \mathrm{O} 10$ & 21.84 & 433.11347 & & 415.1030 & 397.0922 & 379.0818 & 313.0710 & 283.0605 & 10.27 & [18] \\
\hline 19 & Apigenin-C-hexoside-C-pentoside isomer 5 & $\mathrm{C} 26 \mathrm{H} 28 \mathrm{O} 14$ & 22.13 & 565.15574 & & 529.1351 & 397.0923 & 379.0818 & 325.0710 & 295.0606 & 0.29 & \\
\hline $20^{1}$ & Vitexin-2"-O-rhamnoside & $\mathrm{C} 27 \mathrm{H} 30 \mathrm{O} 14$ & 22.21 & 579.17138 & & 433.1135 & 415.1028 & 397.0924 & 313.0709 & 283.0604 & 5.06 & \\
\hline 21 & Apigenin-C-hexoside-C-pentoside isomer 6 & $\mathrm{C} 26 \mathrm{H} 28 \mathrm{O} 14$ & 22.39 & 565.15574 & & 529.1352 & 397.0924 & 379.0815 & 325.0710 & 295.0604 & 0.35 & \\
\hline 22 & Methoxy-tetrahydroxy(iso)flavone-O-hexoside isomer 1 & $\mathrm{C} 22 \mathrm{H} 22 \mathrm{O} 12$ & 22.47 & & 477.10331 & 462.0812 & 315.0516 & 300.0280 & 299.0205 & 271.0252 & 0.07 & \\
\hline 23 & Apigenin-C-hexoside-C-pentoside isomer 7 & $\mathrm{C} 26 \mathrm{H} 28 \mathrm{O} 14$ & 22.61 & 565.15574 & & 529.1351 & 379.0818 & 337.0707 & 325.0710 & 295.0603 & 0.22 & \\
\hline 24 & Isovitexin (Apigenin-6-C-glucoside) & $\mathrm{C} 21 \mathrm{H} 20 \mathrm{O} 10$ & 22.73 & 433.11347 & & 415.1032 & 397.0920 & 379.0815 & 313.0709 & 283.0604 & 26.73 & \\
\hline 25 & Isoscoparin or Scoparin & $\mathrm{C} 22 \mathrm{H} 22 \mathrm{O} 11$ & 23.17 & 463.12404 & & 445.1141 & 427.1030 & 367.0817 & 343.0816 & 313.0711 & 0.18 & \\
\hline 26 & Methoxy-tetrahydroxy(iso)flavone-O-hexoside isomer 2 & $\mathrm{C} 22 \mathrm{H} 22 \mathrm{O} 12$ & 23.36 & & 477.10331 & 462.0811 & 315.0516 & 300.0280 & 299.0201 & 271.0252 & 0.42 & \\
\hline $27^{1}$ & $\begin{array}{l}\text { Isoquercitrin (Quercetin-3-O-glucoside) } \\
\text { (a) }\end{array}$ & $\mathrm{C} 21 \mathrm{H} 20 \mathrm{O} 12$ & 23.47 & & 463.08765 & 301.0358 & 300.0280 & 271.0252 & 255.0300 & 178.9980 & 0.20 & \\
\hline $28^{1}$ & $\begin{array}{l}\text { Isorhamnetin-3-O-glucoside } \\
\text { Istide }\end{array}$ & $\mathrm{C} 22 \mathrm{H} 22 \mathrm{O} 12$ & 25.49 & & 477.10330 & 315.0515 & 314.0437 & 285.0410 & 271.0252 & 243.0298 & 0.27 & \\
\hline 29 & Isorhamnetin-O-hexoside & $\mathrm{C} 22 \mathrm{H} 22 \mathrm{O} 12$ & 25.71 & & 477.10330 & 315.0517 & 314.0436 & 300.0281 & 271.0253 & 243.0298 & 0.19 & \\
\hline 30 & Abscisic acid & $\mathrm{C} 15 \mathrm{H} 20 \mathrm{O} 4$ & 25.81 & & 263.12834 & 219.1388 & 204.1152 & 201.1280 & 152.0832 & 151.0754 & 0.09 & \\
\hline 31 & Dihydroxy(iso)flavone-C-hexoside & $\mathrm{C} 21 \mathrm{H} 20 \mathrm{O} 9$ & 26.19 & 417.11856 & & 399.1074 & 351.0864 & 321.0760 & 297.0761 & 267.0653 & 0.18 & \\
\hline 32 & Methoxy-pentahydroxy(iso)flavone isomer 1 & $\mathrm{C} 16 \mathrm{H} 12 \mathrm{O} 8$ & 26.35 & & 331.04540 & 316.0228 & 287.0203 & 271.0253 & 270.0175 & 259.0247 & 0.74 & \\
\hline 33 & Methoxy-tetrahydroxy(iso)flavone-O-hexoside isomer 3 & $\mathrm{C} 22 \mathrm{H} 22 \mathrm{O} 12$ & 27.20 & & 477.10331 & 462.0810 & 315.0516 & 300.0280 & 299.0205 & 271.0252 & 0.13 & \\
\hline 34 & Methoxy-pentahydroxy(iso)flavone isomer 2 & $\mathrm{C} 16 \mathrm{H} 12 \mathrm{O} 8$ & 27.54 & & 331.04540 & 316.0228 & 287.0192 & 271.0265 & 270.0181 & 259.0237 & 0.40 & \\
\hline $35^{1}$ & Quercetin $\left(3,3^{\prime}, 4^{\prime}, 5,7-\right.$ Pentahydroxyflavone) & $\mathrm{C} 15 \mathrm{H} 10 \mathrm{O} 7$ & 27.57 & & 301.03483 & 273.0410 & 178.9978 & 151.0026 & 121.0282 & 107.0126 & 0.46 & \\
\hline $36^{1}$ & Naringenin (4',5,7-Trihydroxyflavanone) & $\mathrm{C} 15 \mathrm{H} 12 \mathrm{O} 5$ & 27.76 & & 271.06065 & 227.0713 & 177.0184 & 151.0026 & 119.0489 & 107.0126 & 0.19 & \\
\hline 37 & Methoxy-pentahydroxy(iso)flavone isomer 3 & $\mathrm{C} 16 \mathrm{H} 12 \mathrm{O} 8$ & 27.77 & & 331.04540 & 316.0229 & 299.0206 & 287.0214 & 271.0254 & 259.0259 & 1.26 & \\
\hline 38 & Trihydroxy-trimethoxy(iso)flavone-O hexoside & $\mathrm{C} 24 \mathrm{H} 26 \mathrm{O} 13$ & 27.95 & & 521.12952 & 506.1118 & 359.0777 & 358.0700 & 344.0542 & 329.0309 & 0.55 & \\
\hline $39^{1}$ & Luteolin $\left(3^{\prime}, 4^{\prime}, 5,7-\right.$ Tetrahydroxyflavone) & C15H10O6 & 28.44 & & 285.03991 & 217.0506 & 199.0390 & 175.0392 & 151.0027 & 133.0282 & 2.88 & [18] \\
\hline 40 & Quercetin-4'-O-methyl ether & $\mathrm{C} 16 \mathrm{H} 12 \mathrm{O} 7$ & 28.80 & & 315.05048 & 300.0280 & 271.0252 & 255.0300 & 243.0297 & 227.0341 & 8.52 & [18] \\
\hline 41 & Apigenin $\left(4^{\prime}, 5,7\right.$-Trihydroxyflavone $)$ & $\mathrm{C} 15 \mathrm{H} 10 \mathrm{O} 5$ & 30.28 & & 269.04500 & 227.0346 & 225.0553 & 151.0026 & 149.0234 & 117.0331 & 1.51 & [18] \\
\hline 42 & Chrysoeriol (3'-Methoxy-4, $4^{\prime}, 7$-trihydroxyflavone) & $\mathrm{C} 16 \mathrm{H} 12 \mathrm{O} 6$ & 30.59 & & 299.05556 & 284.0330 & 256.0380 & 227.0334 & 151.0025 & 107.0125 & 1.03 & [18] \\
\hline 43 & Dimethoxy-trihydroxy(iso)flavone isomer 1 & $\mathrm{C} 17 \mathrm{H} 14 \mathrm{O} 7$ & 31.11 & & 329.06613 & 314.0438 & 299.0201 & 285.0410 & 271.0252 & 243.0298 & 8.04 & \\
\hline 44 & Dihydroxy-dimethoxy(iso)flavone isomer 1 & $\mathrm{C} 17 \mathrm{H} 14 \mathrm{O} 6$ & 32.37 & & 313.07122 & 298.0487 & 297.0409 & 283.0252 & 269.0457 & 255.0299 & 0.04 & [35] \\
\hline 45 & Dihydroxy-dimethoxy(iso)flavone isomer 2 & C17H14O6 & 32.93 & & 313.07122 & 298.0487 & 297.0408 & 283.0253 & 269.0455 & 255.0301 & 0.07 & [35] \\
\hline 46 & Dimethoxy-trihydroxy(iso)flavone isomer 2 & C17H14O7 & 33.29 & & 329.06613 & 314.0437 & 299.0201 & 285.0407 & 271.0252 & 243.0294 & 8.11 & \\
\hline 47 & Dihydroxy-dimethoxy(iso)flavone isomer 3 & C17H14O6 & 34.82 & & 313.07122 & 298.0487 & 283.0255 & 270.0537 & 269.0461 & 255.0300 & 0.11 & [35] \\
\hline 48 & Dihydroxy-dimethoxy(iso)flavone isomer 4 & $\mathrm{C} 17 \mathrm{H} 14 \mathrm{O} 6$ & $\begin{array}{l}54.02 \\
35.50\end{array}$ & & 313.07122 & 298.0488 & 283.0254 & 269.0465 & 255.0301 & 242.0224 & 0.22 & [35] \\
\hline
\end{tabular}


Among the compounds, luteolin and apigenin and several of their derivatives could be identified. Indeed, apigenin has been reported to possess various beneficial health effects such as antioxidant, anti-inflammatory, and chemopreventive effects [36]. Similarly, luteolin and its glycosides are widely distributed in the plant kingdom and preclinical studies have demonstrated this flavone to exhibit a range of pharmacological actions, including antimicrobial, anticancer, antioxidant and anti-inflammatory [37]. Naringenin was also detected in the extracts. Interestingly, naringenin is endowed with a wide range of biological effects on human health, including antidiabetic, antiviral, cardioprotective, antioxidant, and anti-hyperlipidemic properties, amongst others [38]. Nonetheless, quercetin, also identified in the studied extracts, is a flavonoid found in fruits and vegetables, having unique biological properties that may improve both mental and physical performance and reduce the risk of infections [39]. Epidemiological evidence suggests that flavonoids may play a vital function in the decreased risk of chronic diseases associated with a diet rich in plant-derived foods [37]. Hence, the chemical profiles of extracts of $P$. foetida identified in the present study suggest that the plant can be a good source of flavonoids and therefore can be considered for the potential development of nutraceuticals.

Assays based upon the use of DPPH and ABTS radicals are among the most popular spectrophotometric methods for determining the antioxidant capacity of various samples, including plant extracts [40]. In the present study, all tested extracts were found to be fairly good radical scavengers. However, in DPPH assay, the methanolic (80\%) extract showed the highest scavenging potential, while in ABTS assay, the ethyl acetate extract was the most potent $(68.98 \pm 2.80 \mathrm{mg} / \mathrm{g})$, followed by infusion and water maceration extracts $(62.08 \pm 1.11$ and $61.81 \pm 1.44 \mathrm{mg} / \mathrm{g}$ respectively).

Likewise, ethyl acetate extracts displayed the highest reducing activity in both CUPRAC and FRAP assays (162.83 \pm 0.24 and $65.35 \pm 0.57 \mathrm{mg} / \mathrm{g}$ respectively), followed by methanolic extract (CUPRAC: $103.00 \pm 1.93 \mathrm{mg} / \mathrm{g}$ and FRAP: $35.76 \pm 1.43 \mathrm{mg} / \mathrm{g}$ ). Nonetheless, reducing activity was also reasonably displayed by the other extracts ranging from $54.46-66.83 \mathrm{mg} / \mathrm{g}$ in CUPRAC assay and $30.36-32.38 \mathrm{mg} / \mathrm{g}$ in FRAP assay. Moreover, all extracts acted as metal chelators (14.10-19.21 mg/g), although the highest metal chelating effects were achieved by methanolic and infusion extracts (19.21 \pm 0.09 and $18.00 \pm 0.12 \mathrm{mg} / \mathrm{g}$ respectively). Regarding the phosphomolybdenum assay, the extracts' antioxidant capacity ranged from $0.66-2.68 \mathrm{mM} / \mathrm{g}$, with ethyl acetate extract showing the highest whilst methanolic $(80 \%)$ extract, the lowest activity (Table 4$)$.

Table 4. Antioxidant abilities of the tested extracts.

\begin{tabular}{|c|c|c|c|c|c|c|}
\hline Extracts & DPPH & ABTS & CUPRAC & FRAP & $\begin{array}{c}\text { Metal Chelating } \\
\text { (mg EDTAE/g) }\end{array}$ & $\begin{array}{c}\text { PBD } \\
(\mathrm{mM} \text { TE/g) }\end{array}$ \\
\hline EA & $26.69 \pm 1.85^{b}$ & $68.98 \pm 2.80^{a}$ & $162.83 \pm 0.24^{\mathrm{a}}$ & $65.35 \pm 0.57^{a}$ & $15.60 \pm 0.26^{c}$ & $2.68 \pm 0.19^{a}$ \\
\hline $\mathrm{MeOH}(80 \%)$ & $31.74 \pm 0.90^{a}$ & $55.06 \pm 3.32^{c}$ & $66.83 \pm 0.21^{\mathrm{c}}$ & $30.36 \pm 0.48^{\mathrm{d}}$ & $15.24 \pm 0.58^{c}$ & $0.66 \pm 0.10^{d}$ \\
\hline Water maceration & $20.77 \pm 0.18^{c}$ & $61.81 \pm 1.44^{b}$ & $54.46 \pm 1.44^{\mathrm{e}}$ & $31.59 \pm 0.28^{c, d}$ & $14.10 \pm 0.40^{\mathrm{d}}$ & $1.01 \pm 0.01^{\mathrm{c}}$ \\
\hline
\end{tabular}

Values are reported as mean \pm S.D. EA: Ethyl acetate; $\mathrm{MeOH}$ : methanolic; TE: Trolox equivalent; EDTAE: EDTA equivalent; PBD: Phosphomolybdenum. Different letters (a, b, c, d, and e) indicate significant differences in the extracts $(p<0.05)$.

Other studies have also attested to the antioxidant potential of $P$. foetida extract by the radical scavenging mechanism. For instance, Ajane and Patil [30] reported P. foetida extract to show scavenging ability in both DPPH $\left(\mathrm{IC}_{50}: 614.405 \mu \mathrm{g} / \mathrm{mL}\right)$ and ABTS $\left(\mathrm{IC}_{50}: 25.18 \mu \mathrm{g} / \mathrm{mL}\right)$ assays. Moreover, in the same study, P. foetida leaves were found to possess total antioxidant potential comparable to ascorbic acid. The antioxidant potential also correlated with the extract content in phenols, flavonoids, and saponins [30]. Hence, the relatively higher antioxidant potential of ethyl acetate extract obtained in the present study could be attributed to the comparatively higher phenolic content present. Indeed, phenolic compounds may exert their antioxidant activity in different ways [41]. 
Furthermore, high antiradical properties $\left(\mathrm{EC}_{50}\right.$ of $\left.1.37 \pm 1.17 \mu \mathrm{g} / \mathrm{mL}\right)$ and moderate antioxidant reducing power $(0.41 \pm 0.03 \mathrm{mM} \mathrm{FE})$, with $82.09 \pm 13.82 \mathrm{mg}$ gallic acid equivalent $/ \mathrm{g}$ of TPC and $205.59 \pm 6.57 \mathrm{mg}$ quercetin equivalent/g of TFC values were displayed by $P$. foetida methanolic extract [29]. Similarly, reducing power and free radical scavenging potential was demonstrated by fruit and leaf extracts of $P$. foetida. However, the antioxidant activity of the leaf extract was higher than the fruit extract. Additionally, phytochemicals such as alkaloids, phenolics, flavonoids, saponins, and cardiac glycosides were found in the leaf extract [42].

Cholinesterase (ChE) inhibitors are a significant target in the treatment of Alzheimer's disease [43]. On the pharmacy shelf, some drugs such as tacrine and rivastigmine have been produced as cholinesterase inhibitors for alleviating Alzheimer's disease. As a result, a number of botanicals used in various traditional systems of medicines as memory enhancers have been evaluated for anti-cholinesterase activity [44]. In the present investigation, with the exception of ethyl acetate extract which was found to inhibit BChE selectively $(2.45 \pm 0.18 \mathrm{mg}$ GALAE/g), P. foetida extracts inhibited AChE selectively. Particularly, the methanolic extract was observed to be the most potent AChE inhibitor $(2.22 \pm 0.04 \mathrm{mg}$ GALAE/g), while water maceration, methanolic $(80 \%)$ and infusion extracts showed moderate inhibition against AChE (0.50-0.92 mg GALAE/g) (Table 5). Previous studies have also reported the AChE inhibition induced by P. foetida extract [45]. An infusion was also reported as a moderate inhibitor in our previous paper [46].

Table 5. Enzyme inhibitory properties of the tested extracts.

\begin{tabular}{|c|c|c|c|c|c|}
\hline \multirow{2}{*}{ Extracts } & AChE & BChE & \multirow{2}{*}{$\begin{array}{c}\text { Tyrosinase } \\
\text { (mg KAE/g) }\end{array}$} & Amylase & Glucosidase \\
\hline & \multicolumn{2}{|c|}{ (mg GALAE/g) } & & \multicolumn{2}{|c|}{ (mM ACAE/g) } \\
\hline EA & na & $2.45 \pm 0.18$ & $48.48 \pm 3.68^{a}$ & $0.59 \pm 0.01^{a}$ & $0.70 \pm 0.01^{a}$ \\
\hline $\mathrm{MeOH}$ & $2.22 \pm 0.04^{\mathrm{a}}$ & na & $29.33 \pm 1.71^{b}$ & $0.43 \pm 0.03^{b}$ & $0.37 \pm 0.01^{b}$ \\
\hline $\mathrm{MeOH}(80 \%)$ & $0.92 \pm 0.02^{b}$ & na & $35.11 \pm 5.77^{b}$ & $0.35 \pm 0.02^{c}$ & $0.30 \pm 0.01^{c}$ \\
\hline Water maceration & $0.77 \pm 0.08^{c}$ & na & na & $0.17 \pm 0.01^{\mathrm{d}}$ & $0.11 \pm 0.01^{\mathrm{d}}$ \\
\hline Infusion & $0.50 \pm 0.08^{\mathrm{d}}$ & na & na & $0.41 \pm 0.01^{b}$ & $0.28 \pm 0.01^{c}$ \\
\hline
\end{tabular}

Values are reported as mean \pm S.D. EA: Ethyl acetate; GALAE: Galantamine equivalent; KAE: Kojic acid equivalent; ACAE: Acarbose equivalent. na: not active. Different letters $(a, b, c, d)$ indicate significant differences in the extracts $(p<0.05)$.

Tyrosinase is a key enzyme in the synthesis of melanin, thus it can be regarded as the main target for controlling hyperpigmentation problems [47]. In the present study, only the ethyl acetate and the two methanolic extracts showed anti-tyrosinase inhibitory potentials, whereas water maceration and infusion extracts were both inactive against tyrosinase. Ethyl acetate extract exhibited the highest anti-tyrosinase activity $(48.48 \pm 3.68 \mathrm{mg} \mathrm{KAE} / \mathrm{g})$, followed by the methanolic extracts $(29.33 \pm 1.71$ and $35.11 \pm 5.77 \mathrm{mg} \mathrm{KAE} / \mathrm{g}$ ) (Table 5). Interestingly, in addition to inhibition of melanin formation in B16 melanoma cells and the exhibition of DPPH radical-scavenging activity, P. foetida was also found to inhibit tyrosinase enzyme [48]. It is noteworthy that antioxidants have been reported to exhibit potent inhibitory activities towards tyrosinase and melanin production and have consequently been used to inhibit melanogenesis [49].

In fact, a high correlation was obtained between mushroom tyrosinase inhibition and ABTS and DPPH radical scavenging activity in previous reports suggesting that free radical scavenging activity enhanced the inhibition of mushroom tyrosinase activity [50]. Hence, the relatively good anti-tyrosinase activity of the extracts reported in the present work also could be ascribed to their respective antioxidant potency.

Chronic hyperglycemia has been considered as one of the principal causes of several diabetic complications. Since the major source of glucose is dietary carbohydrates, the inhibition of key carbohydrates digesting enzymes, such as $\alpha$-amylase and $\alpha$-glucosidase, can be vital in preventing a postprandial rise in blood glucose, chronic hyperglycemia and hence diabetic complications. This is because inhibitors of these enzymes ( $\alpha$-amylase and $\alpha$-glucosidase) delay the carbohydrate digestion 
and reduce the rate of glucose absorption from the gut, lowering the postprandial rise in blood glucose level. Therefore, inhibition of $\alpha$-amylase and $\alpha$-glucosidase is key in the management and treatment of non-insulin-dependent diabetes mellitus or type 2 diabetes [51,52].

Interestingly, in the present study, all tested extracts demonstrated dual inhibition against $\alpha$-amylase $(0.17-0.59 \mathrm{mM} / \mathrm{g})$ and $\alpha$-glucosidase $(0.11-0.70 \mathrm{mM} / \mathrm{g})$. However, the ethyl acetate extract displayed the highest anti-amylase $(0.59 \pm 0.01 \mathrm{mM} / \mathrm{g})$ and anti-glucosidase $(0.70 \pm 0.01 \mathrm{mM} / \mathrm{g})$ effects. Comparatively, water maceration extract showed the least activity against these enzymes $(0.17 \pm 0.01$ and $0.11 \pm 0.01 \mathrm{mM} / \mathrm{g}$ respectively). Nonetheless, the other tested extracts displayed moderate inhibitory potentials $\alpha$-amylase and $\alpha$-glucosidase (Table 5).

Remarkably, the findings of the present study were in agreement with previous studies which also reported the antidiabetic potential of $P$. foetida by inhibiting the enzymes involved in postprandial hyperglycemia. Maximum $\alpha$-amylase inhibitory activity was recorded in $100 \mu \mathrm{g} / \mathrm{mL}$ of aqueous and ethanolic extract of root with inhibition of $80.3 \%$ and $83.3 \%$, respectively. In the $\alpha$-glucosidase inhibitory activity, the aqueous extract of seed of $P$. foetida showed maximum inhibition of $72.3 \%$, followed by the ethanolic extract of its root which demonstrated inhibition of $65.7 \%$ [53]. Likewise, several other Passiflora species have been reported to be potent inhibitors of $\alpha$-amylase and $\alpha$-glucosidase. This was largely attributed to their high phenolic/flavonoid content and antioxidant activity [54,55]. Recent studies have also established polyphenols rich plants to have the ability to chelate the enzymes $\alpha$-amylase and $\alpha$-glucosidase through alteration in a structure that is coupled with the loss of biological functions [54,56].

Based on the results of HPLC-MS fingerprint analysis, in silico, in vitro, and ex vivo studies were conducted with the aim to unravel putative pharmacokinetics, target proteins and toxicological effects underlying $P$. foetida extract rational use in phytotherapy. Formerly, the brine shrimp toxicological assay was conducted with the aim to define the biocompatibility limits of methanol and water extracts, in the range $1-20 \mathrm{mg} / \mathrm{mL}$. The tests yielded $\mathrm{LC}_{50}$ values $<5 \mathrm{mg} / \mathrm{mL}$ for both $P$. foetida extracts. These data were also confirmed by in silico toxicological evaluation conducted through the "Toxicity Estimation Software Tool" (T.E.S.T.) developed by the United States Environmental Protection Agency (EPA). Specifically, the analyses were conducted on selected phytochemicals, namely apigenin, chrysoeriol, isovitexin, loliolide, luteolin, quercetin, and vitexin.

The in silico eco-toxicological tests were conducted on the Daphnia magna model that yielded $\mathrm{LC}_{50}$ values in the range $2-26 \mathrm{mg} / \mathrm{mL}$. This is consistent, albeit partially, with the experimental $\mathrm{LC}_{50}$ values obtained with the brine shrimp test. The selected phytochemicals were also analyzed through the bioinformatics platforms SwissADME and SwissTargetPrediction, as well, with the aim to predict pharmacokinetics and putative targets, respectively. The results of the bioinformatics approach are fully reported in the Supplementary Materials (Bioinformatics Folder), whereas the synthetic components-targets analysis (Network Pharmacology) is included in Figure 1.

All these compounds were predicted to be absorbed through the gastrointestinal tract, as well, whereas the sole loliolide was predicted to cross the blood brain barrier (BBB). Among all proteins identified by the bioinformatics analysis, we focused our attention on selected enzymes, namely AChE, cyclo-oxygenase (COX)-2, tyrosinase, and monoamine oxidase (MAO)-A. The selected enzymes were docked with apigenin, chrysoeriol, isovitexin, loliolide, luteolin, quercetin, and vitexin, in order to confirm the bioinformatics predictions and calculate putative affinity constants $(\mathrm{Ki})$ that were in the submicromolar-micromolar range $(0.2-10.5 \mu \mathrm{M})$ (Figure $2 \mathrm{~A}-\mathrm{O})$. 


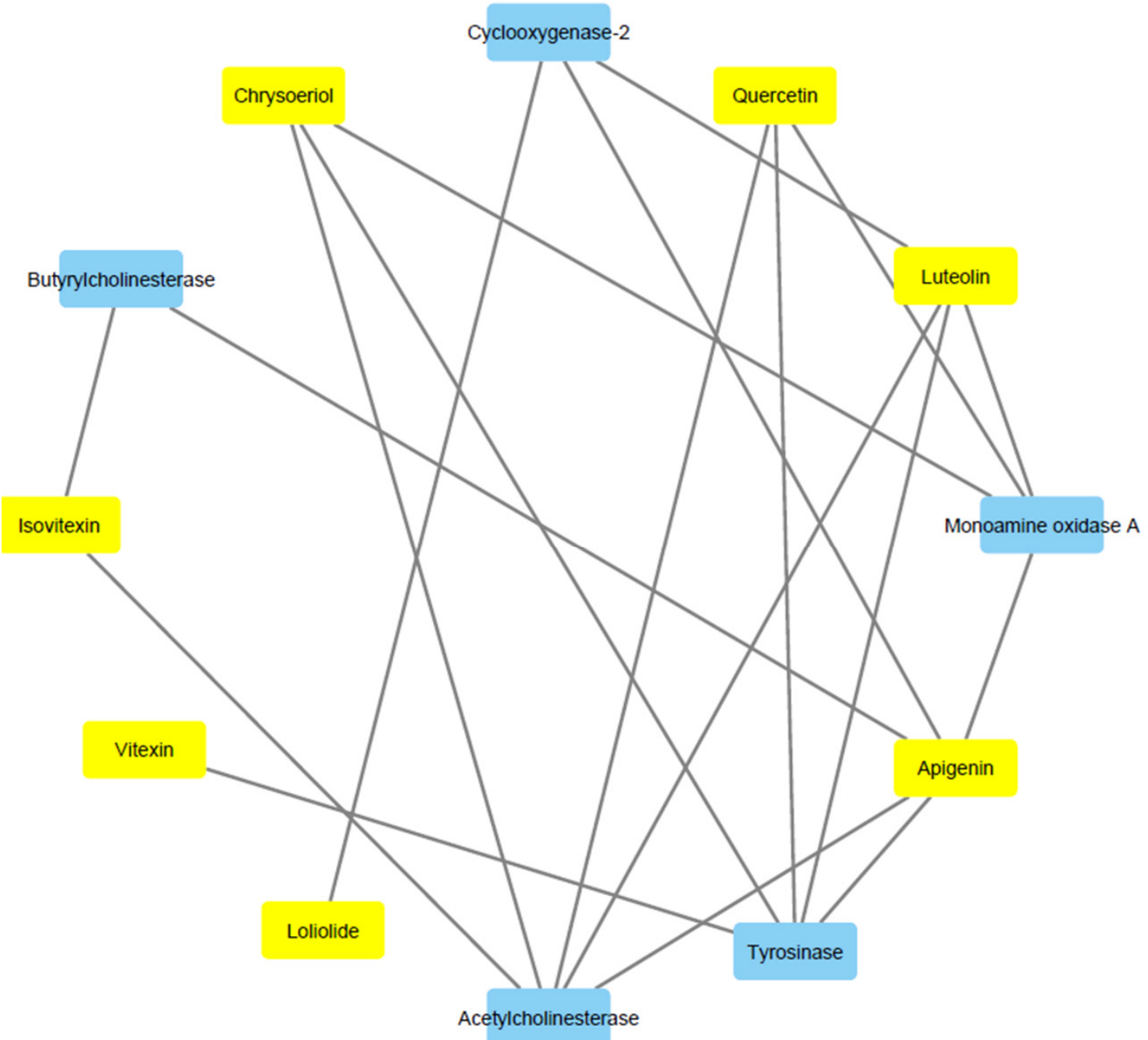

Figure 1. Components-Targets analysis. 

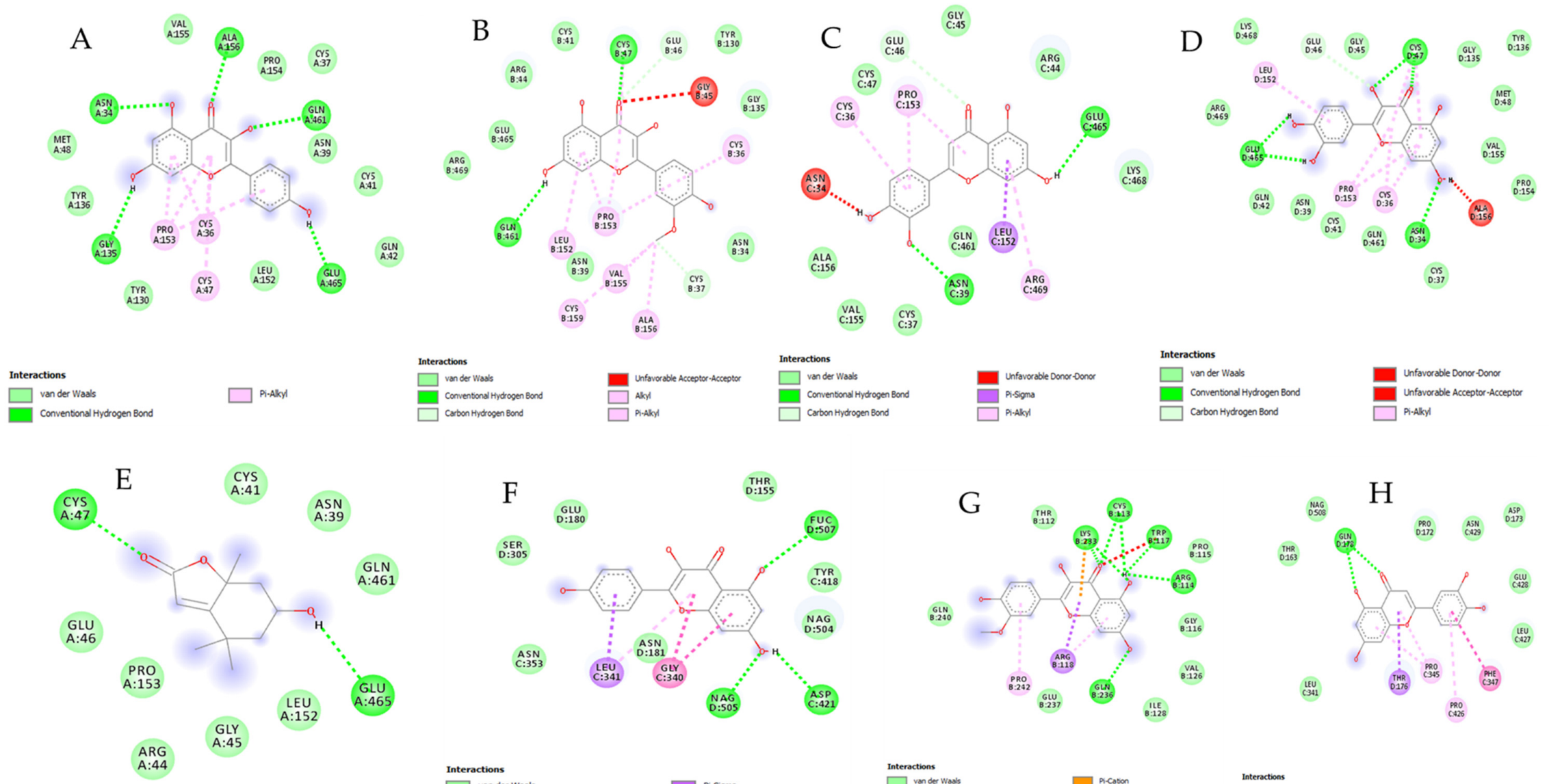

Interactions
$\square$ vander Wasts
(a)
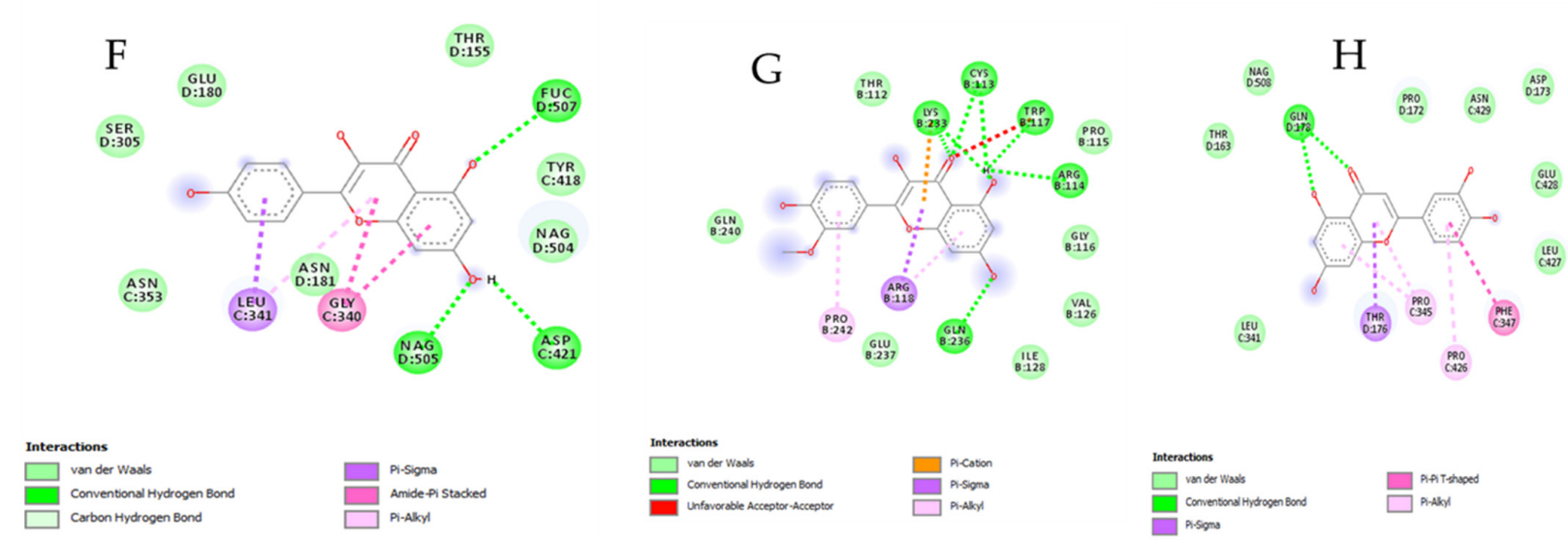

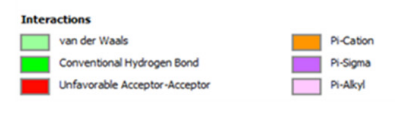

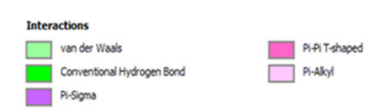

Figure 2. Cont. 


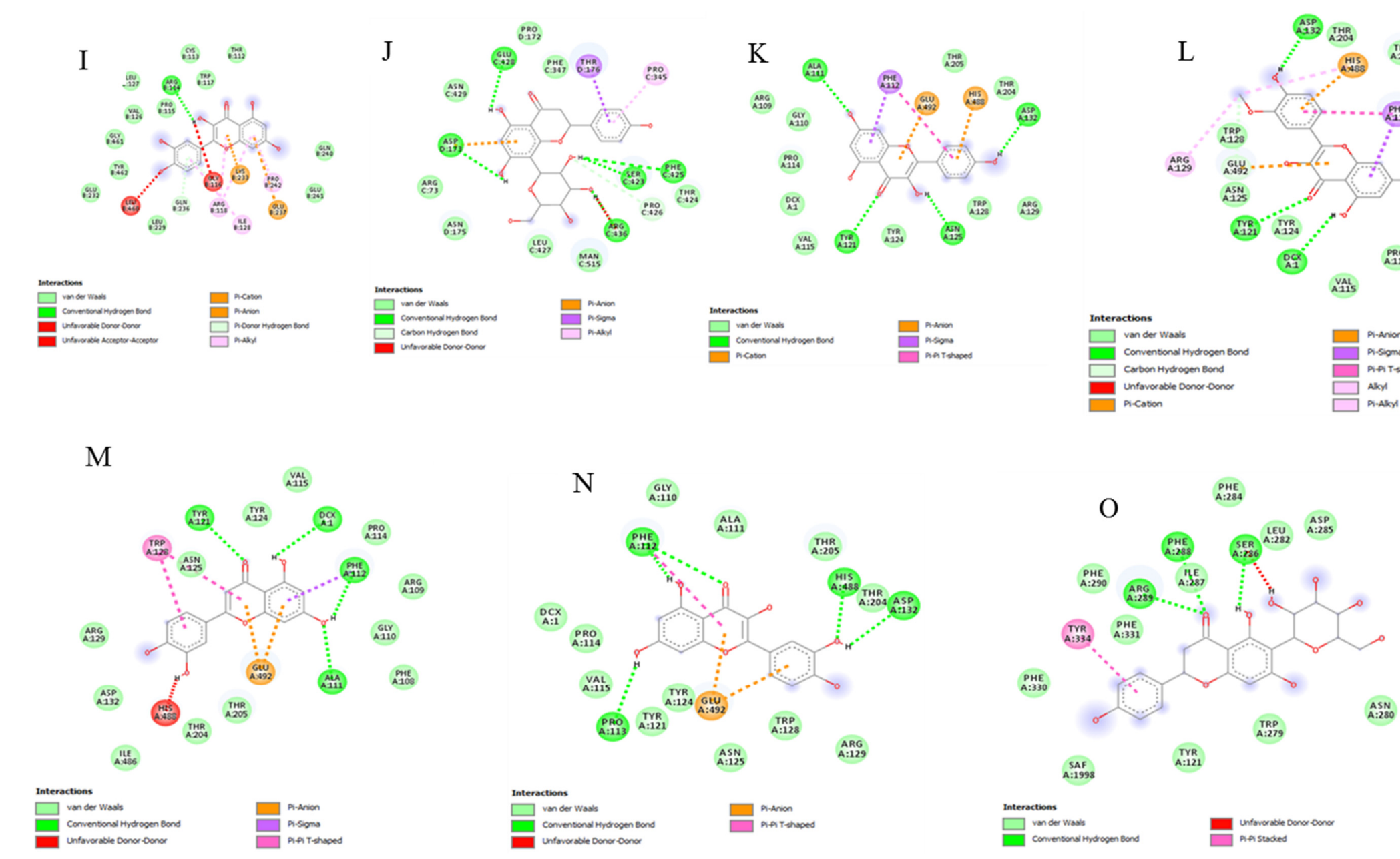

Figure 2. (A): Putative interaction between apigenin and COX-2 (PDB: 1CX2). Free energy of binding $(\Delta \mathrm{G})$ and affinity (Ki) are $-8.8 \mathrm{kcal} / \mathrm{mol}$ and $0.4 \mu \mathrm{M}$, respectively; (B): Putative interaction between chrysoeriol and COX-2 (PDB: 1CX2). Free energy of binding $(\Delta \mathrm{G})$ and affinity (Ki) are $-9.3 \mathrm{kcal} / \mathrm{mol}$ and $0.2 \mu \mathrm{M}$, respectively; (C): Putative interaction between luteolin and COX-2 (PDB: 1CX2). Free energy of binding $(\Delta \mathrm{G})$ and affinity (Ki) are $-9.5 \mathrm{kcal} / \mathrm{mol}$ and $0.1 \mu \mathrm{M}$, respectively; (D): Putative interaction between quercetin and COX-2 (PDB: 1CX2). Free energy of binding $(\Delta \mathrm{G})$ and affinity (Ki) are $-9.3 \mathrm{kcal} / \mathrm{mol}$ and $0.2 \mu \mathrm{M}$, respectively; (E): Putative interaction between loliolide and COX-2 (PDB: 1CX2). Free energy of binding $(\Delta \mathrm{G})$ and affinity (Ki) are $-6.8 \mathrm{kcal} / \mathrm{mol}$ and $10.5 \mu \mathrm{M}$, respectively; 
(F): Putative interaction between apigenin and tyrosinase (PDB: 5M8P). Free energy of binding $(\Delta \mathrm{G})$ and affinity (Ki) are $-8.1 \mathrm{kcal} / \mathrm{mol}$ and $1.2 \mu \mathrm{M}$, respectively; (G): Putative interaction between chrysoeriol and tyrosinase (PDB: 5M8P). Free energy of binding $(\Delta \mathrm{G})$ and affinity (Ki) are $-7.8 \mathrm{kcal} / \mathrm{mol}$ and $1.9 \mu \mathrm{M}$, respectively;

(H): Putative interaction between luteolin and tyrosinase (PDB: $5 \mathrm{M} 8 \mathrm{P})$. Free energy of binding $(\Delta \mathrm{G})$ and affinity (Ki) are $-8.2 \mathrm{kcal} / \mathrm{mol}$ and $0.9 \mu \mathrm{M}$, respectively;

(I): Putative interaction between quercetin and tyrosinase (PDB: $5 \mathrm{M} 8 \mathrm{P})$. Free energy of binding $(\Delta \mathrm{G})$ and affinity $(\mathrm{Ki})$ are $-8.3 \mathrm{kcal} / \mathrm{mol}$ and $0.8 \mu \mathrm{M}$, respectively;

(J): Putative interaction between vitexin and tyrosinase (PDB: $5 \mathrm{M} 8 \mathrm{P})$. Free energy of binding $(\Delta \mathrm{G})$ and affinity (Ki) are $-8.6 \mathrm{kcal} / \mathrm{mol}$ and $0.5 \mu \mathrm{M}$, respectively;

(K): Putative interaction between apigenin and MAO-A (PDB: 2Z5X). Free energy of binding $(\Delta \mathrm{G})$ and affinity (Ki) are $-8.2 \mathrm{kcal} / \mathrm{mol}$ and $0.9 \mu \mathrm{M}$, respectively;

(L): Putative interaction between chrysoeriol and MAO-A (PDB: $2 Z 5 X)$. Free energy of binding $(\Delta \mathrm{G})$ and affinity (Ki) are $-8.3 \mathrm{kcal} / \mathrm{mol}$ and $0.8 \mu \mathrm{M}$, respectively;

(M): Putative interaction between luteolin and MAO-A (PDB: 2Z5X). Free energy of binding $(\Delta \mathrm{G})$ and affinity (Ki) are $-8.4 \mathrm{kcal} / \mathrm{mol}$ and $0.7 \mu \mathrm{M}$, respectively;

(N): Putative interaction between quercetin and MAO-A (PDB: 2Z5X). Free energy of binding $(\Delta \mathrm{G})$ and affinity (Ki) are $-8.3 \mathrm{kcal} / \mathrm{mol}$ and $0.8 \mu \mathrm{M}$, respectively;

(O): Putative interaction between quercetin and AchE (PDB: 1GQR). Free energy of binding $(\Delta \mathrm{G})$ and affinity $(\mathrm{Ki})$ are $-8.7 \mathrm{kcal} / \mathrm{mol}$ and $0.4 \mu \mathrm{M}$, respectively [57]. 
The putative interactions between extracts' phytochemicals and selected enzymes were also confirmed, albeit partially, by the aforementioned colorimetric tests and the pharmacological paradigms discussed below. In this regard, methanol and water extracts were studied through in vitro and ex vivo models in order to confirm their efficacy in experimental paradigms mimicking the burden of oxidative stress and inflammation at both central and peripheral levels. Specifically, hypothalamic HypoE22 cells were treated with scalar extract concentrations $(10-500 \mu \mathrm{g} / \mathrm{mL})$, with the highest concentration being at least 10 -fold lower than the $\mathrm{LC}_{50}$ calculated via brine shrimp test [22]. As evidenced by the MTT viability test, the methanol and water extracts were tolerated by HypoE22 cells, in the aforementioned concentration range, in both basal and hydrogen peroxide-induced oxidative stress conditions (Figure 3). Additionally, the lowest tested concentration of the water extract induced a mild stimulation of the cell viability, as well.

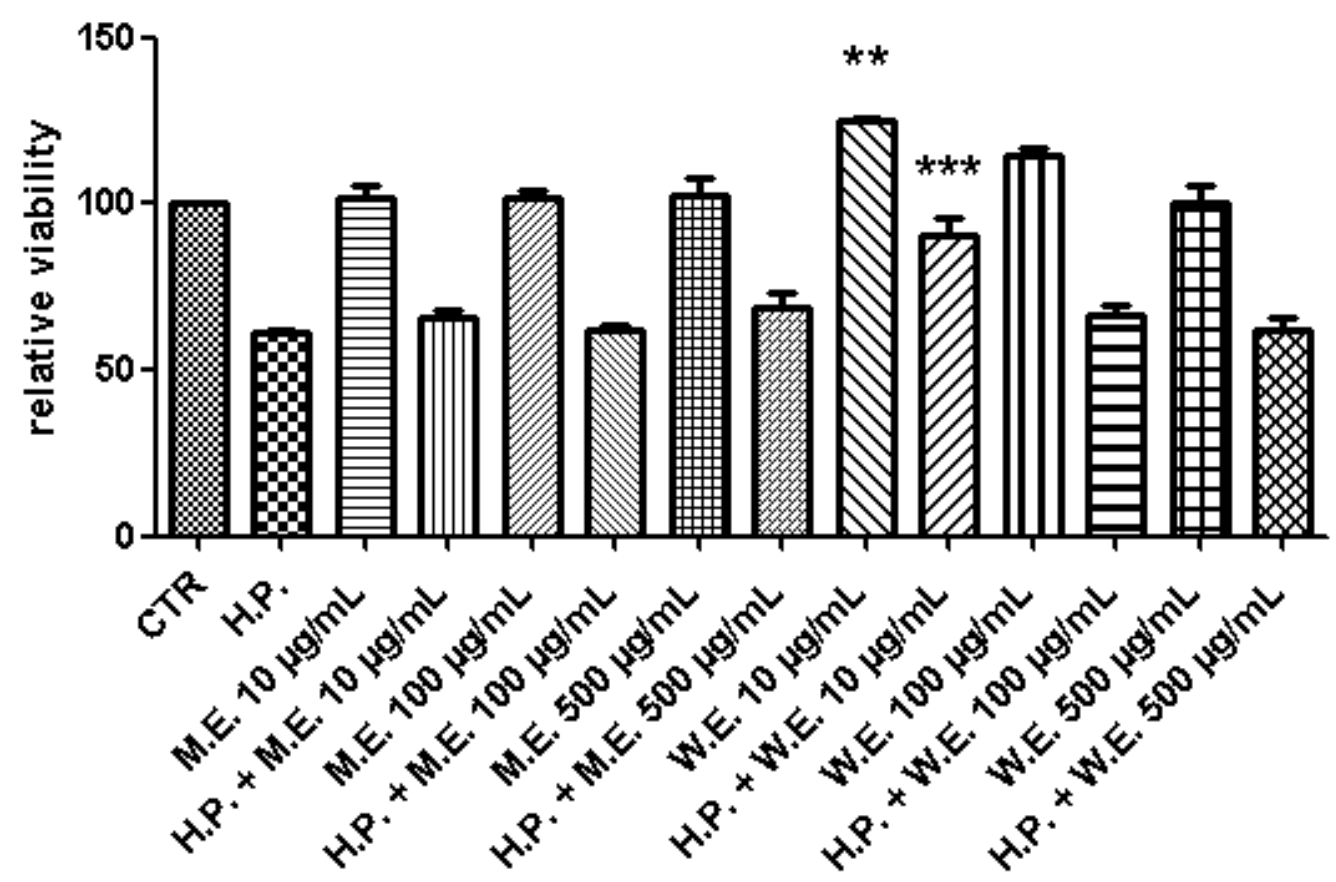

Figure 3. Effects of $P$. foetida methanol (M.E.) and water (W.E.) extracts on basal and hydrogen peroxide $300 \mu \mathrm{M}$ (H.P.)-induced hypothalamic HypoE22 cell viability (MTT test). Cell viability was relatively calculated towards the untreated control (CTR) group. Data were analyzed through analysis of variance (ANOVA), followed by post hoc Newman-Keuls test. ANOVA, $p<0.001$; ${ }^{* *} p<0.01$ vs. H.P; *** $p<0.001$.

By contrast, the wound healing test showed the water extract-induced decrease in the spontaneous migration (expressed as percentage of scratch-free area) of HypoE22 cells, in the $24 \mathrm{~h}$ following the experimental lesion of cell monolayer (Figure $4 \mathrm{~A}, \mathrm{~B}$ ). This suggests a minor role exerted by extract as a neuroprotective agent.

Additionally, none of the two extracts were able to prevent the hydrogen peroxide-induced decrease of extracellular 5-HT level (Figure 5). 


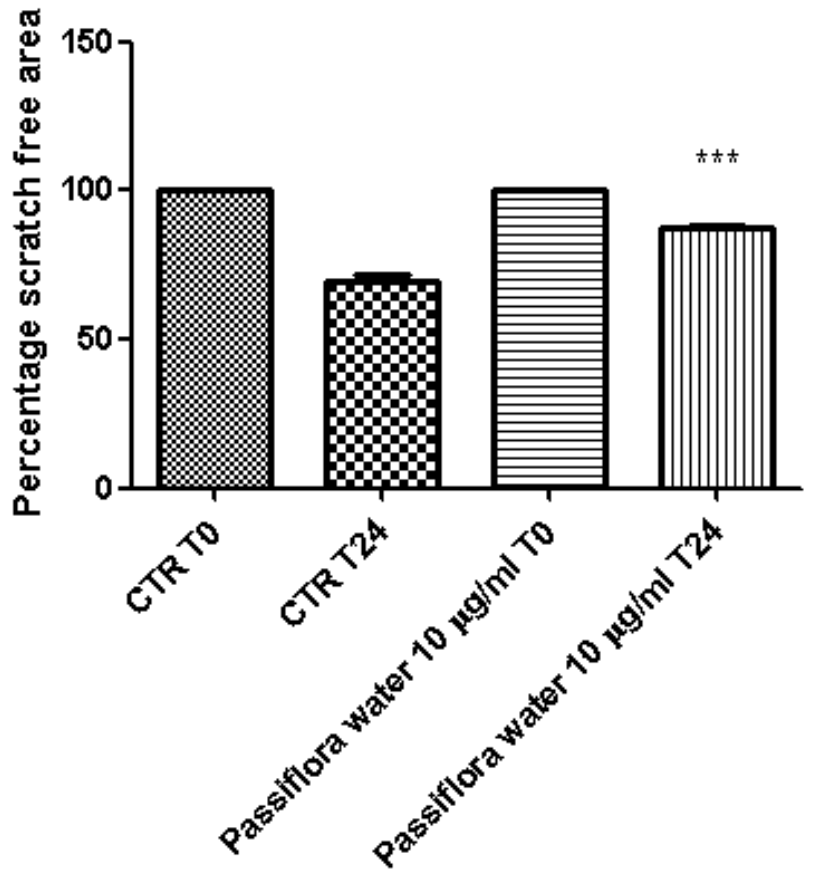

(A)

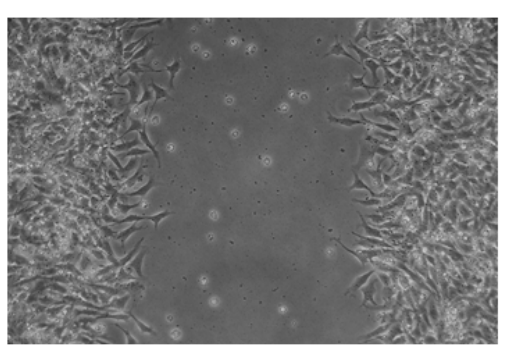

CTR: TO

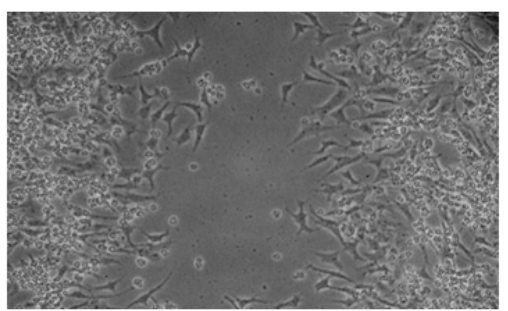

CTR: T24

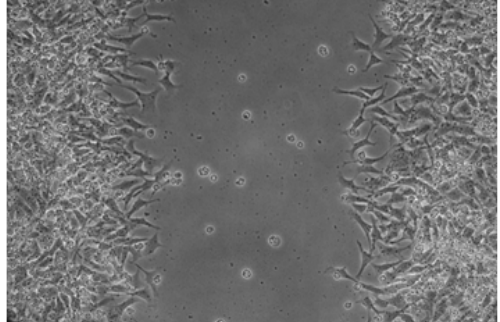

W.E. $10 \mu \mathrm{g} / \mathrm{mL}$ : TO

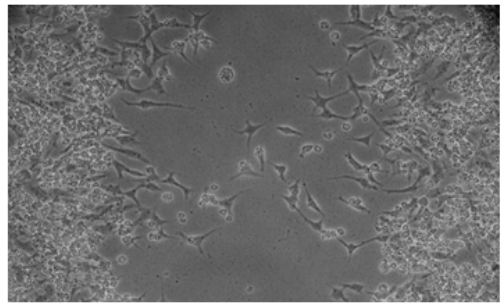

W.E. $10 \mu \mathrm{g} / \mathrm{mL}$ : T24

(B)

Figure 4. Effects of subtoxic concentration $(10 \mu \mathrm{g} / \mathrm{mL})$ of $P$. foetida water extract on spontaneous hypothalamic HypoE22 cell migration. Quantification of free cell area (A) and representative images (B) of wound healing test recorded at different time (T) points: 0 and $24 \mathrm{~h}$ following stimulation. ANOVA, $p<0.001 ;{ }^{* * *} p<0.001$ vs. CTR T24. 


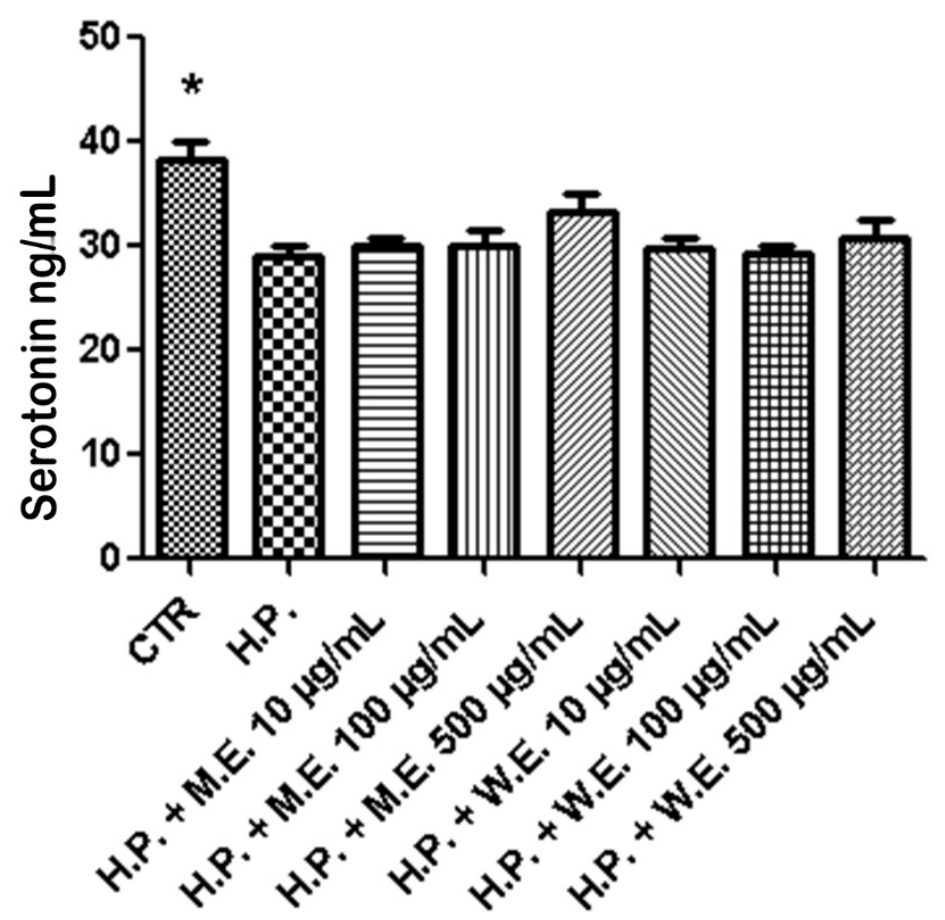

Figure 5. Effects of $P$. foetida methanol (M.E.) and water (W.E.) extracts on hydrogen peroxide $300 \mu \mathrm{M}$ (H.P.)-induced decrease of serotonin (5-HT) release from hypothalamic HypoE22 cell. 5-HT release was quantified through HPLC coupled to coulometric detection and expressed as ng/mL. Data were analyzed through analysis of variance (ANOVA), followed by post hoc Newman-Keuls test. ANOVA, $p<0.05 ;{ }^{*} p<0.05$ vs. H.P.

As a consequence, the putative MAO-A inhibition, at least in part predicted by docking calculations, is questionable. MAO-A is deeply involved in 5-HT catabolism, and increased oxidative stress and inflammatory conditions in the brain are able to upregulate MAO-A activity [58,59]. Although most of the selected phytochemicals showed good affinities towards MAO-A, the two extracts were ineffective in preventing hydrogen-peroxide depletion of hypothalamic 5-HT. Additionally, none of these phytochemicals was predicted to cross $\mathrm{BBB}$, with the exception of the sole loliolide that was found to interact with the COX-2. In this context, we can hypothesize that the traditional uses of P. foetida in treating neurological and psychiatric disorders [9] could derive, at least partially, from the potential efficacy of plant-deriving phytochemicals in counteracting the inflammatory components underlying these chronic diseases [60].

P. foetida was also reported to exert protective effects in skin inflammatory models [8]. Based on the aforementioned anti-tyrosinase effects induced by the extracts, and further confirmed by docking calculations on apigenin, chrysoeriol, luteolin, quercetin, and vitexin, we conducted an ex vivo study in order to verify the efficacy of $P$. foetida water and methanol extracts on isolated mouse skin specimens challenged with hydrogen peroxide $(1 \mathrm{mM})$, that, besides functioning as a classical oxidative stress stimulus, is also able to increase tyrosinase activity [61]. The increased tyrosinase activity was monitored through the evaluation of the skin L-dopa level [62], compared to the untreated control (CTR) group (Figure 6). By contrast, the extract efficacy was evaluated as the prevention of hydrogen peroxide-induced L-dopa turnover. Both extracts were able to prevent L-dopa turnover, thus further confirming the anti-tyrosinase activity suggested by both colorimetric and docking analyses. 


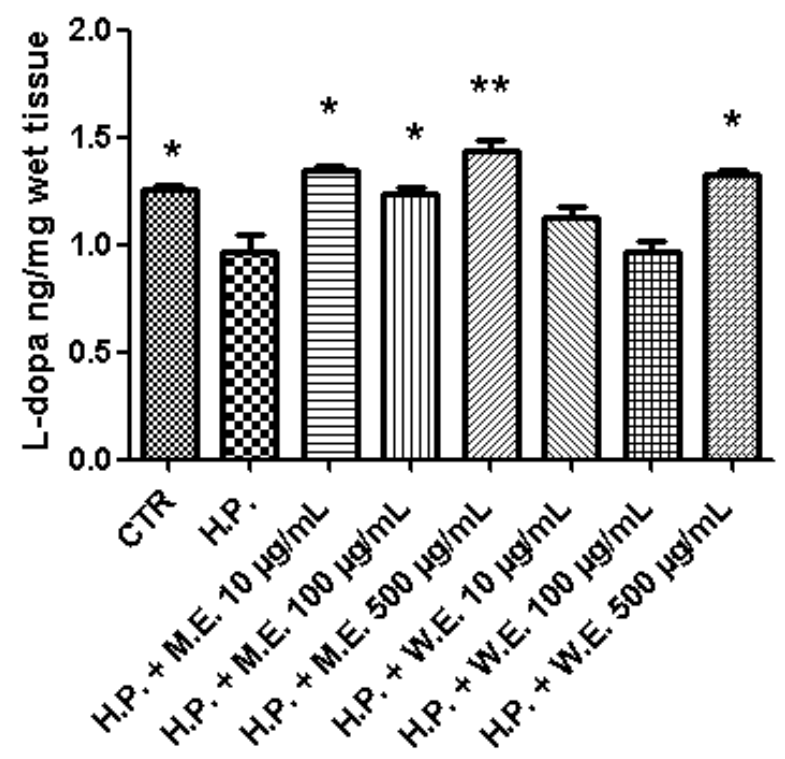

Figure 6. Effects of $P$. foetida methanol (M.E.) and water (W.E.) extracts on hydrogen peroxide $1 \mathrm{mM}$ (H.P.)-induced decrease of 1-dopa release from isolated mouse skin. 1-dopa release was quantified through HPLC coupled to coulometric detection and expressed as $\mathrm{ng} / \mathrm{mg}$ wet tissue. Data were analyzed through analysis of variance (ANOVA), followed by post hoc Newman-Keuls test. ANOVA, $p<0.001{ }^{*} p<0.05,{ }^{* *} p<0.01$ vs. H.P.

Additionally, the extracts were also effective in blunting hydrogen peroxide-induced level of $\mathrm{PGE}_{2}$ (Figure 7). This also agrees with the calculated affinity of apigenin, chrysoeriol, luteolin, quercetin, and loliolide towards COX-2.

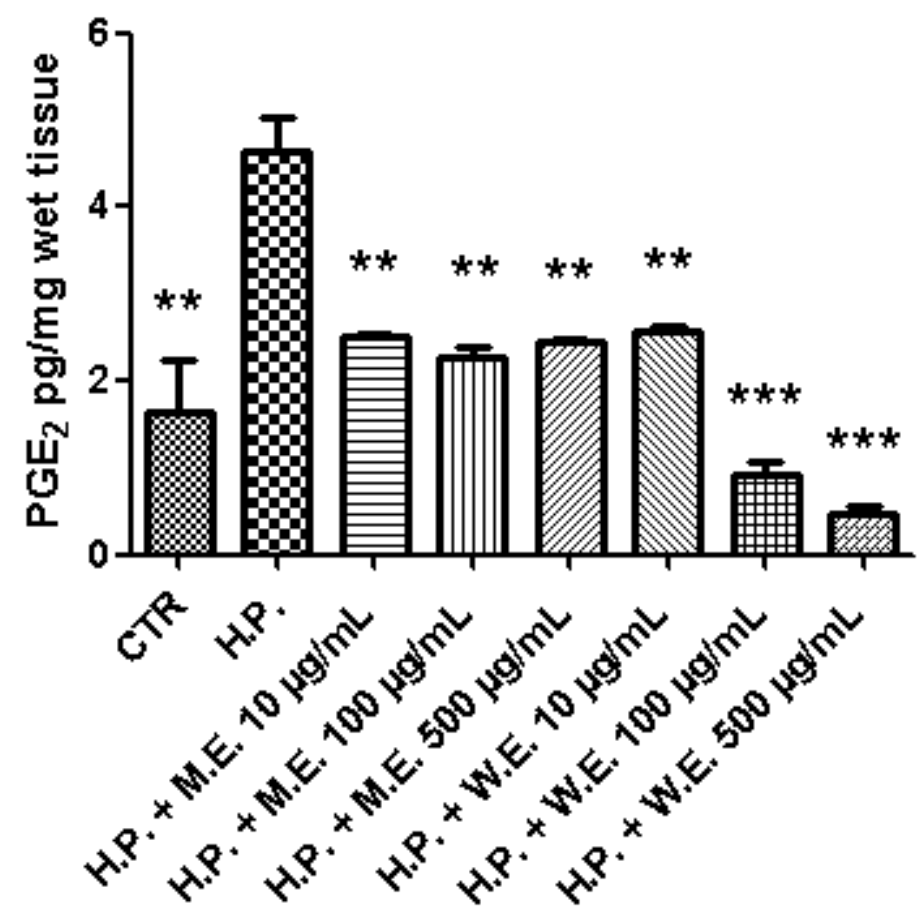

Figure 7. Effects of $P$. foetida methanol (M.E.) and water (W.E.) extracts on hydrogen peroxide $1 \mathrm{mM}$ (H.P.)-induced decrease of $\mathrm{PGE}_{2}$ release from isolated mouse skin. ANOVA, $p<0.001 ;{ }^{* *} p<0.01$, *** $p<0.001$ vs. H.P. 
Finally, considering the potential antimicrobial applications of $P$. foetida [1], methanol and water extracts were also tested against multiple dermatophytes strains, namely Tricophyton and Aerthrodrma species (Table 6), that are involved in skin disorders, including hyperpigmentation [63-65]. Both extracts were able to inhibit fungal growth and these results add to the aforementioned inhibitory activity against tyrosinase, which is crucial in the pharmacotherapy of hyperpigmentation. The present antimycotic activity could be related, at least partially, to the extract phenol and flavonoid content of P. foetida [66] and further supports the phytotherapy use of $P$. foetida as a skin protective agent.

Table 6. Minimal inhibitory concentrations (MICs) of $P$ foetida methanol and water extracts, and griseofulvin towards selected dermatophytes.

\begin{tabular}{cccc}
\hline \multirow{2}{*}{ Dermatophytes (ID strain) A3:D15 } & \multicolumn{3}{c}{ Minimum Inhibitory Concentration (MIC) } \\
\cline { 2 - 4 } & $\begin{array}{c}\text { Methaol Extract } \\
\left(\boldsymbol{\mu g} \mathbf{~ m L}^{-\mathbf{1}}\right)^{*}\end{array}$ & $\begin{array}{c}\text { Water Extract } \\
\left(\boldsymbol{\mu g} \mathbf{~ m L}^{-\mathbf{1}}\right)^{*}\end{array}$ & $\begin{array}{c}\text { Griseofulvin } \\
\left(\boldsymbol{\mu g} \mathbf{~ m L}^{-\mathbf{1}}\right)^{*}\end{array}$ \\
\hline Arthroderma crocatum (CCF 5300) & $12.4(7.81-15.625)$ & $39.37(31.25-62.5)$ & $>8$ \\
Arthroderma curreyi (CCF 5207) & $6.19(3.9-7.81)$ & $12.4(7.81-15.625)$ & $>8$ \\
Arthroderma gypseum (CCF 6261) & $157.49(125-250)$ & $396(250-500)$ & $1.587(1-2)$ \\
Arthroderma insingulare (CCF 5417) & $12.4(7.81-15.625)$ & $19.68(15.625-31.25)$ & $>8$ \\
Arthroderma quadrifidum (CCF 5792) & $78.74(62.5-125)$ & $314.98(250-500)$ & $>8$ \\
Trichophyton mentagrophytes (CCF 4823) & $49.6(31.25-62.5)$ & $99.21(62.5-125)$ & $2.52(2-4)$ \\
Trichophyton mentagrophytes (CCF 5930) & $157.49(125-250)$ & $396(250-500)$ & $3.174(2-4)$ \\
Trichophyton rubrum (CCF 4933) & $39.37(31.25-62.5)$ & $99.21(62.5-125)$ & $1.26(1-2)$ \\
Trichophyton rubrum (CCF 4879) & $99.21(62.5-125)$ & $198,42(125-250)$ & $3.175(2-4)$ \\
Trichophyton tonsurans (CCF 4834) & $9.84(7.81-15.625)$ & $9.84(7.81-15.625)$ & $0.198(0.125-0.25)$ \\
\hline
\end{tabular}

${ }^{*}$ MIC values are reported as geometric means of three independent replicates $(n=3)$; MIC ranges are reported within brackets. MIC values are reported as $<$ [lowest concentration tested].

\section{Conclusions}

Passiflora species have been greatly appraised for their vast range of bioactive compounds and pharmacological properties. In this study, P. foetida extracts prepared using different solvents were found to yield a varying amount of total phenolic and flavonoid contents. Particularly, ethyl acetate and methanolic (80\%) extracts were found to contain the highest phenolic and flavonoid contents respectively. Nonetheless, the infusion and methanolic extracts were both found to be rich in flavonoids with the presence of flavones, flavone glycosides, amongst others. Additionally, P. foetida extracts displayed notable enzyme inhibitory effects by acting as inhibitors of $\alpha$-amylase, $\alpha$-glucosidase, tyrosinase, acetyl- and butyryl-cholinesterase, although the extracts differed in their inhibition capacities. All P. foetida extracts also showed good overall antioxidant potentials by acting as radical scavenging, reducing, and metal chelating mechanisms. The enzyme inhibition and antioxidant effects were also confirmed by both docking and pharmacological evaluations. Finally, the extracts were also effective against multiple dermatophytes strains involved in skin inflammation. Hence, the results obtained from the current investigation evidently support the therapeutic properties of $P$. foetida as antioxidant, antidiabetic, and anti-hyperpigmentation agents and thus could help to stimulate further consideration to understand their potential influence on human health as potential pharmaceuticals and nutraceuticals.

Supplementary Materials: The following are available online at http://www.mdpi.com/2227-9717/8/9/1034/s1, Supplementary Folder.

Author Contributions: Conceptualization, C.F., P.A., G.O., G.Z., L.M.; methodology, C.F, P.A., G.O., G.Z., L.M.; software, C.F., G.Z.; validation, C.F., P.A., G.O., G.Z., L.M.; formal analysis, C.F., P.A., G.O., G.Z., L.M.; investigation, A.C., S.C.D.S., M.C.C., K.I.S., G.A.F., L.R., S.L., R.V., O.K.E., G.A., S.J., J.J., Z.C., C.F., P.A., G.O., G.Z., L.M.; data curation, C.F., P.A., G.O., G.Z., L.M.; writing—original draft preparation, M.F.M.; writing-review and editing, C.F., P.A., G.O., G.Z., L.M., M.F.M.; visualization, L.B.; supervision, L.B.; project administration, C.F., G.O., L.M.; funding acquisition, C.F., G.O., L.M. All authors have read and agreed to the published version of the manuscript.

Funding: The study was supported by funds from Cristalfarma S.r.l. (Milan, Italy) within joint projects coordinated by Giustino Orlando, Luigi Menghini and Claudio Ferrante. 
Conflicts of Interest: The authors declare no conflict of interest.

\section{References}

1. Simão, M.J.; Barboza, T.J.; Vianna, M.G.; Garcia, R.D.O.; Mansur, E.; Ignacio, A.C.P.; Pacheco, G. A comparative study of phytoconstituents and antibacterial activity of in vitro derived materials of four Passiflora species. Anais da Academia Brasileira de Ciências 2018, 90, 2805-2813. [CrossRef] [PubMed]

2. Casierra-Posada, F.; Jarma-Orozco, A. Nutritional Composition of Passiflora Species. In Nutritional Composition of Fruit Cultivars; Elsevier BV: Amsterdam, The Netherlands, 2016; pp. 517-534.

3. Gadioli, I.L.; Cunha, M.D.S.B.D.; De Carvalho, M.V.O.; Costa, A.M.; Pineli, L.D.L.D.O. A systematic review on phenolic compounds inPassifloraplants: Exploring biodiversity for food, nutrition, and popular medicine. Crit. Rev. Food Sci. Nutr. 2017, 58, 785-807. [CrossRef] [PubMed]

4. Dhawan, K.; Dhawan, S.; Sharma, A. Passiflora: A review update. J. Ethnopharmacol. 2004, 94, 1-23. [CrossRef] [PubMed]

5. $\quad$ Chen, Y.-Z.; Wei, X.; Li, M.; Duan, X.; Sun, Y.-M.; Yang, R.-L.; Su, X.-D.; Huang, R.; Wang, H. Nutritional Composition and Antioxidant Properties of the Fruits of a Chinese Wild Passiflora foetida. Molecules 2018, 23, 459. [CrossRef]

6. Ramaiya, S.D.; Bujang, J.S.; Zakaria, M.H.; King, W.S.; Sahrir, M.A.S. Sugars, ascorbic acid, total phenolic content and total antioxidant activity in passion fruit (Passiflora) cultivars. J. Sci. Food Agric. 2012, 93, 1198-1205. [CrossRef]

7. Revathy, S.; Sunilkumar, T.C. Phytochemical and nutritional studies on the fruit pulp extract of Passiflora foetida Linn. J. Pharmacogn. Phytochem. 2019, 8, 732-734.

8. Mohanasundari, C.; Natarajan, D.; Srinivasan, K.; Umamaheswari, S.; Ramachandran, A. Antibacterial properties of Passiflora foetida L. a common exotic medicinal plant. Afr. J. Biotechnol. 2007, 6, 2650-2653. [CrossRef]

9. Patil, A.S.; Paikrao, H.M.; Patil, S.R. Passiflora foetida Linn: A complete morphological and phytopharmacological review. Int J Pharma. Bio. Sci. 2013, 4, 285-296.

10. Sasikala, V.; Saravanan, S.; Parimelazhagan, T. Analgesic and anti-inflammatory activities of Passiflora foetida L. Asian Pac. J. Trop. Med. 2011, 4, 600-603. [CrossRef]

11. Sutar, V.; Bhosale, U.P. Screening of Passiflora foetida extracts as anticancer agents on MCF 7 cell line. BIOINFOLET-A 2013, 10, 808-810.

12. Anandan, R.; Jayakar, B.; Jeganathan, S.; Manavalan, R.; Kumar, S.R. Effect of ethanol extract of fruits of Passiflora foetida Linn. on CCl. J. Pharm. Res. 2009, 2, 413-415.

13. Rahman, M.A.; Hossain, M.A.; Hasan, M.S.; Hossain, M.G. Antinociceptive, antidiarrhoeal and cytotoxic activities of Passiflora foetida linn. Pharmacologyonline 2011, 1, 228-236.

14. Santosh, P.; Venugopl, R.; Nilakash, S.; Kunjbihari, S.; Mangala, L. Antidepressant activity of methanolic extract of Passiflora foetida leaves in mice. Int. J. Pharm. Pharm. Sci. 2011, 3, 112-115.

15. Asadujjaman, M.; Mishuk, A.U.; Hossain, A.M.; Karmakar, U.K. Medicinal potential of Passiflora foetida L. plant extracts: Biological and pharmacological activities. J. Integr. Med. 2014, 12, 121-126. [CrossRef]

16. Sathish, R.; Sahu, A.; Natarajan, K. Antiulcer and antioxidant activity of ethanolic extract of Passiflora foetida L. Indian J. Pharmacol. 2011, 43, 336-339. [CrossRef] [PubMed]

17. Ranganatha, N.; Kuppast, D.I.J.; Veerashekar, T. Study of Anti-Hypertension Activity of Aerial Parts of Passiflora foetida Linn. Int. Res. J. Pharm. Plant Sci. 2013, 1, 1-12.

18. Nguyen, T.Y.; To, D.C.; Tran, M.H.; Lee, J.S.; Lee, J.-H.; Kim, J.A.; Woo, M.H.; Min, B.S. Anti-inflammatory Flavonoids Isolated from Passiflora foetida. Nat. Prod. Commun. 2015, 10, 929-931. [CrossRef]

19. Dall'Acqua, S.; Aktumsek, A. Investigation of Antioxidant Potentials of Solvent Extracts From Different Anatomical Parts of Asphodeline Anatolica E. Tuzlaci: An Endemic Plant to Turkey. Afr. J. Tradit. Complement. Altern. Med. 2014, 11, 481-488. [CrossRef]

20. Dall'Acqua, S.; Uysal, A.; Diuzheva, A.; Gunes, E.; Jekő, J.; Cziáky, Z.; Picot-Allain, C.M.N.; Mahomoodally, F.M. Characterization of phytochemical components of Ferula halophila extracts using HPLC-MS/MS and their pharmacological potentials: A multi-functional insight. J. Pharm. Biomed. Anal. 2018, 160, 374-382. [CrossRef] 
21. Grochowski, D.; Uysal, S.; Aktumsek, A.; Granica, S.; Dall'Acqua, S.; Ceylan, R.; Locatelli, M.; Tomczyk, M. In vitro enzyme inhibitory properties, antioxidant activities, and phytochemical profile of Potentilla thuringiaca. Phytochem. Lett. 2017, 20, 365-372. [CrossRef]

22. Ferrante, C.; Recinella, L.; Ronci, M.; Menghini, L.; Brunetti, L.; Chiavaroli, A.; Leone, S.; Di Iorio, L.; Carradori, S.; Tirillini, B.; et al. Multiple pharmacognostic characterization on hemp commercial cultivars: Focus on inflorescence water extract activity. Food Chem. Toxicol. 2019, 125, 452-461. [CrossRef] [PubMed]

23. Menghini, L.; Ferrante, C.; Leporini, L.; Recinella, L.; Chiavaroli, A.; Leone, S.; Pintore, G.; Vacca, M.; Orlando, G.; Brunetti, L. An Hydroalcoholic Chamomile Extract Modulates Inflammatory and Immune Response in HT29 Cells and Isolated Rat Colon. Phytotherapy Res. 2016, 30, 1513-1518. [CrossRef] [PubMed]

24. Iodice, P.; Ferrante, C.; Brunetti, L.; Cabib, S.; Protasi, F.; Walton, M.E.; Pezzulo, G. Fatigue modulates dopamine availability and promotes flexible choice reversals during decision making. Sci. Rep. 2017, 7, 535. [CrossRef] [PubMed]

25. Gu, L.; Lu, J.; Li, Q.; Wu, N.; Zhang, L.; Li, H.; Xing, W.; Zhang, X. A network-based analysis of key pharmacological pathways of Andrographis paniculata acting on Alzheimer's disease and experimental validation. J. Ethnopharmacol. 2020, 251, 112488. [CrossRef]

26. Chiavaroli, A.; Sinan, K.I.; Zengin, G.; Mahomoodally, F.M.; Sadeer, N.B.; Etienne, O.K.; Cziáky, Z.; Jekő, J.; Glamočlija, J.; Sokovic, M.; et al. Identification of Chemical Profiles and Biological Properties of Rhizophora racemosa G. Mey. Extracts Obtained by Different Methods and Solvents. Antioxidants 2020, 9, 533. [CrossRef]

27. Van Ngo, T.; Scarlett, C.J.; Bowyer, M.C.; Ngo, P.D.; Van Vuong, Q. Impact of Different Extraction Solvents on Bioactive Compounds and Antioxidant Capacity from the Root of Salacia chinensis L. J. Food Qual. 2017, 2017, 1-8. [CrossRef]

28. Asir, P.J.; Priyanga, S.; Hemmalakshmi, S.; Devaki, K. In Vitro Free Radical Scavenging Activity and Secondary Metabolites in Passiflora foetida L. Asian. J. Pharmaceut. Res. Health Care. 2014, 6, 3-11.

29. Sisin, N.N.T.; Abdullah, H.; Sul'ain, M.D. Antiproliferative, antioxidative and compounds identification from methanolic extract of Passiflora foetida and its fractions. J. Anal. Pharm. Res. 2017, 6, 00166. [CrossRef]

30. Ajane, G.; Patil, A.S. Evaluation of Antioxidant Potential of Passiflora foetida Extract and Quantitative Evaluation of its Phytochemical Content-A Possible Natural Antioxidant. Pharm. Chem. J. 2019, 6, 14-24.

31. Ulrih, N.P.; Capanoglu, E.; Jassbi, A.R.; Miron, A. Advance on the Flavonoid C-glycosides and Health Benefits. Crit. Rev. Food Sci. Nutr. 2015, 56, S29-S45. [CrossRef]

32. Zucolotto, S.M.; Fagundes, C.; Reginatto, F.H.; Ramos, F.A.; Castellanos, L.; Duque, C.; Schenkel, E.P. Analysis of C -glycosyl Flavonoids from South American Passiflora Species by HPLC-DAD and HPLC-MS. Phytochem. Anal. 2011, 23, 232-239. [CrossRef] [PubMed]

33. Simirgiotis, M.; Schmeda-Hirschmann, G.; Borquez, J.; Kennelly, E.J. The Passiflora tripartita (Banana Passion) Fruit: A Source of Bioactive Flavonoid C-Glycosides Isolated by HSCCC and Characterized by HPLC-DAD-ESI/MS/MS. Molecules 2013, 18, 1672-1692. [CrossRef] [PubMed]

34. Dall'Acqua, S.; Mahomoodally, F.M.; Paksoy, M.Y.; Picot-Allain, C.; Glamocilja, J.; Sokovic, M.; Diuzheva, A.; Jekő, J.; Cziáky, Z.; Rodrigues, M.J.; et al. Phytochemical characterization and bioactivities of five Apiaceae species: Natural sources for novel ingredients. Ind. Crop. Prod. 2019, 135, 107-121. [CrossRef]

35. Echeverri, F.; Cardona, G.; Torres, F.; Peláez, C.; Quiñones, W.; Renteria, E. Ermanin: An insect deterrent flavonoid from Passiflora foetida resin. Phytochem. 1991, 30, 153-155. [CrossRef]

36. Shukla, R.; Pandey, V.; Vadnere, G.P.; Lodhi, S. Role of Flavonoids in Management of Inflammatory Disorders. In Bioactive Food as Dietary Interventions for Arthritis and Related Inflammatory Diseases, 2nd ed.; Watson, R.R., Preedy, V.R., Eds.; Elsevier: London, UK, 2019; pp. 293-322.

37. López-Lázaro, M. Distribution and biological activities of the flavonoid luteolin. Mini-Rev. Med. Chem. 2009, 9, 31-59. [CrossRef]

38. Salehi, B.; Fokou, P.V.T.; Sharifi-Rad, M.; Zucca, P.; Pezzani, R.; Martins, N.; Sharifi-Rad, J. The Therapeutic Potential of Naringenin: A Review of Clinical Trials. Pharmaceuticals 2019, 12, 11. [CrossRef]

39. Davis, J.M.; Murphy, E.A.; Carmichael, M.D. Effects of the Dietary Flavonoid Quercetin Upon Performance and Health. Curr. Sports Med. Rep. 2009, 8, 206-213. [CrossRef]

40. Bendini, A.; Cerretani, L.; Pizzolante, L.; Toschi, T.G.; Guzzo, F.; Ceoldo, S.; Marconi, A.M.; Andreetta, F.; Levi, M. Phenol content related to antioxidant and antimicrobial activities of Passiflora spp. extracts. Eur. Food Res. Technol. 2005, 223, 102-109. [CrossRef] 
41. Walter, M.; Marchesan, E. Phenolic compounds and antioxidant activity of rice. Braz. Arch. Boil. Technol. 2011, 54, 371-377. [CrossRef]

42. Tandoro, Y.; Widyawati, P.S.; Budianta, T.D.W.; Sumargo, G. Phytochemical identification and antioxidant activity of passiflora foetida fruits and leaves extracts: A comparative study. Int. J. Pharm. Pharm. Sci. 2020, 12,55-58. [CrossRef]

43. Gauthier, S.; Emre, M.; Farlow, M.R.; Bullock, R.; Grossberg, G.T.; Potkin, S.G. Strategies for continued successful treatment of Alzheimer's disease: Switching cholinesterase inhibitors. Curr. Med Res. Opin. 2003, 19, 707-714. [CrossRef] [PubMed]

44. Ahmed, F.; Ghalib, R.M.; Sasikala, P.; Ahmed, K.K.M. Cholinesterase inhibitors from botanicals. Pharmacogn. Rev. 2013, 7, 121. [CrossRef] [PubMed]

45. Melo Filho, A.A.; Kamezaki, A.K.; Estevam Ribeiro, P.R.; Goncalves Reis De Melo, A.C.; Montero Fernandez, I.; Carvalho Dos Santos, R.; Alves Chagas, E.; Cardoso Chagas, P. Chemical Composition, Antioxidant and Biological Activity of Leaves Passiflora foetida. Chem. Eng. Trans. 2018, 64, 241-246. [CrossRef]

46. Zhang, L.; Rocchetti, G.; Zengin, G.; Ak, G.; Yıldıztugay, E.; Mahomoodally, F.M.; Picot-Allain, M.C.N.; Lucini, L. Profiling of polyphenols and sesquiterpenoids using different extraction methods in Muscari turcicum, an endemic plant from Turkey. Ind. Crop. Prod. 2020, 154, 112626. [CrossRef]

47. Pillaiyar, T.; Manickam, M.; Namasivayam, V. Skin whitening agents: Medicinal chemistry perspective of tyrosinase inhibitors. J. Enzym. Inhib. Med. Chem. 2017, 32, 403-425. [CrossRef]

48. Arung, E.T.; Kusuma, I.W.; Christy, E.O.; Shimizu, K.; Kondo, R. Evaluation of medicinal plants from Central Kalimantan for antimelanogenesis. J. Nat. Med. 2009, 63, 473-480. [CrossRef]

49. Cui, H.-X.; Duan, F.-F.; Jia, S.-S.; Cheng, F.-R.; Yuan, K. Antioxidant and Tyrosinase Inhibitory Activities of Seed Oils from Torreya grandis Fort. ex Lindl. BioMed Res. Int. 2018, 2018, 1-10. [CrossRef]

50. Chatatikun, M.; Supjaroen, P.; Promlat, P.; Chantarangkul, C.; Waranuntakul, S.; Nawarat, J.; Tangpong, J.; Chiabchalard, A. Antioxidant and Tyrosinase Inhibitory Properties of an Aqueous Extract of Garcinia atroviridis Griff. ex. T. Anderson Fruit Pericarps. Pharmacogn. J. 2020, 12, 71-78. [CrossRef]

51. Hullatti, K.; Telagari, M. In-vitro $\alpha$-amylase and $\alpha$-glucosidase inhibitory activity of Adiantum caudatum Linn. and Celosia argentea Linn. extracts and fractions. Indian J. Pharmacol. 2015, 47, 425-429. [CrossRef]

52. Islam, S.; Chipiti, T.; Ibrahim, M.; Singh, M. In vitro $\alpha$-amylase and $\alpha$-glucosidase inhibitory and cytotoxic activities of extracts from Cissus cornifolia planch parts. Pharmacogn. Mag. 2017, 13, 329. [CrossRef]

53. Paulraj, J.A.; Subharamanian, H.; Suriyamoorthy, P.; Kanakasabapathi, D. Phytochemical screening, GC-MS analysis and enzyme inhibitory activity of Passiflora foetida L. Indo Am. J. Pharm. Res. 2014, 4, 3526-3534.

54. Saravanan, S.; Parimelazhagan, T. In vitro antioxidant, antimicrobial and anti-diabetic properties of polyphenols of Passiflora ligularis Juss. fruit pulp. Food Sci. Hum. Wellness 2014, 3, 56-64. [CrossRef]

55. Shanmugam, S.; Gomes, I.A.; Denadai, M.; Lima, B.D.S.; Araújo, A.A.D.S.; Narain, N.; Neta, M.T.S.L.; Serafini, M.R.; Quintans, J.S.; Thangaraj, P. UHPLC-QqQ-MS/MS identification, quantification of polyphenols from Passiflora subpeltata fruit pulp and determination of nutritional, antioxidant, $\alpha$-amylase and $\alpha$-glucosidase key enzymes inhibition properties. Food Res. Int. 2018, 108, 611-620. [CrossRef] [PubMed]

56. Nagappan, H.; Pee, P.P.; Kee, S.H.Y.; Ow, J.T.; Yan, S.W.; Chew, L.Y.; Kong, K.-W. Malaysian brown seaweeds Sargassum siliquosum and Sargassum polycystum: Low density lipoprotein (LDL) oxidation, angiotensin converting enzyme (ACE), $\alpha$-amylase, and $\alpha$-glucosidase inhibition activities. Food Res. Int. 2017, 99, 950-958. [CrossRef]

57. Artuğer, F.; Özkaynak, F. A Novel Method for Performance Improvement of Chaos-Based Substitution Boxes. Symmetry 2020, 12, 571. [CrossRef]

58. Lee, J.J.; Chang, C.K.; Liu, I.M.; Chi, T.C.; Yu, H.-J.; Cheng, J.T. Changes in endogenous monoamines in aged rats. Clin. Exp. Pharmacol. Physiol. 2001, 28, 285-289. [CrossRef]

59. Di Giacomo, V.; Ferrante, C.; Ronci, M.; Cataldi, A.; Di Valerio, V.; Rapino, M.; Recinella, L.; Chiavaroli, A.; Leone, S.; Vladimir-Knežević, S.; et al. Multiple pharmacological and toxicological investigations on Tanacetum parthenium and Salix alba extracts: Focus on potential application as anti-migraine agents. Food Chem. Toxicol. 2019, 133, 110783. [CrossRef]

60. Beheshti, F.; Hashemzehi, M.; Hosseini, M.; Marefati, N.; Memarpour, S. Inducible nitric oxide synthase plays a role in depression- and anxiety-like behaviors chronically induced by lipopolysaccharide in rats: Evidence from inflammation and oxidative stress. Behav. Brain Res. 2020, 392, 112720. [CrossRef] 
61. Karg, E.; Odh, G.; Wittbjer, A.; Rosengren, E.; Rorsman, H. Hydrogen peroxide as an inducer of elevated tyrosinase level in melanoma cells. J. Invest. Dermatol. 1993, 100, 209-2013. [CrossRef]

62. Kim, Y.-J.; Kim, M.-J.; Kweon, D.-K.; Lim, S.-T.; Lee, S.-J. Quantification of Hypopigmentation Activity In Vitro. J. Vis. Exp. 2019, 145, e58185. [CrossRef]

63. Zhang, M.; Jiang, L.; Li, F.; Xu, Y.; Lv, S.; Wang, B. Simultaneous dermatophytosis and keratomycosis caused by Trichophyton interdigitale infection: A case report and literature review. BMC Infect. Dis. 2019, 19, 983-988. [CrossRef] [PubMed]

64. Isa-Isa, R.; Arenas, R.; Isa, M. Inflammatory tinea capitis: Kerion, dermatophytic granuloma, and mycetoma. Clin. Dermatol. 2010, 28, 133-136. [CrossRef] [PubMed]

65. El-Zawawy, N.A.; Ali, S.S. Pyocyanin as anti-tyrosinase and anti tinea corporis: A novel treatment study. Microb. Pathog. 2016, 100, 213-220. [CrossRef] [PubMed]

66. Orlando, G.; Recinella, L.; Chiavaroli, A.; Brunetti, L.; Leone, S.; Carradori, S.; Di Simone, S.; Ciferri, M.C.; Zengin, G.; Ak, G.; et al. Water Extract from Inflorescences of Industrial Hemp Futura 75 Variety as a Source of Anti-Inflammatory, Anti-Proliferative and Antimycotic Agents: Results from In Silico, In Vitro and Ex Vivo Studies. Antioxidants 2020, 9, 437. [CrossRef]

(C) 2020 by the authors. Licensee MDPI, Basel, Switzerland. This article is an open access article distributed under the terms and conditions of the Creative Commons Attribution (CC BY) license (http://creativecommons.org/licenses/by/4.0/). 